ARTICLE OPEN

\title{
AIF-regulated oxidative phosphorylation supports lung cancer development
}

\author{
Shuan Rao ${ }^{1,2}$, Laura Mondragón ${ }^{3,4,5,6}$, Blanka Pranjic ${ }^{2}$, Toshikatsu Hanada², Gautier Stoll $\mathbb{1}^{3,4,5,6,7}$, Thomas Köcher ${ }^{8}$, Peng Zhang ${ }^{9}$, \\ Alexander Jais ${ }^{10}$, Alexander Lercher (D) ${ }^{11}$, Andreas Bergthaler ${ }^{11}$, Daniel Schramek (D) ${ }^{12}$, Katharina Haigh ${ }^{13,14,15}$, Valentina Sica ${ }^{3,4,5,6}$, \\ Marion Leduc ${ }^{3,4,5,6}$, Nazanine Modjtahedi ${ }^{16,17,18}$, Tsung-Pin Pai ${ }^{2}$, Masahiro Onji' ${ }^{2}$, rris Uribesalgo ${ }^{2}$, Reiko Hanada ${ }^{2}$, Ivona Kozieradzki ${ }^{2}$, \\ Rubina Koglgruber ${ }^{2}$, Shane J. Cronin ${ }^{2}$, Zhigang She ${ }^{19}$, Franz Quehenberger $\mathbb{D}^{20}$, Helmut Popper $\mathbb{D}^{21}$, Lukas Kenner $\mathbb{D}^{22,23}$, \\ Jody J. Haigh ${ }^{13,14,15}$, Oliver Kepp $\mathbb{1 0}^{3,4,5,6}$, Malgorzata Rak ${ }^{24}$, Kaican Cai', Guido Kroemer ${ }^{3,4,5,6,25,26,27}$ and Josef M. Penninger $\mathbb{1}^{2,28}$
}

Cancer is a major and still increasing cause of death in humans. Most cancer cells have a fundamentally different metabolic profile from that of normal tissue. This shift away from mitochondrial ATP synthesis via oxidative phosphorylation towards a high rate of glycolysis, termed Warburg effect, has long been recognized as a paradigmatic hallmark of cancer, supporting the increased biosynthetic demands of tumor cells. Here we show that deletion of apoptosis-inducing factor (AIF) in a Kras ${ }^{G 12 D}$-driven mouse lung cancer model resulted in a marked survival advantage, with delayed tumor onset and decreased malignant progression. Mechanistically, Aif deletion leads to oxidative phosphorylation (OXPHOS) deficiency and a switch in cellular metabolism towards glycolysis in non-transformed pneumocytes and at early stages of tumor development. Paradoxically, although Aif-deficient cells exhibited a metabolic Warburg profile, this bioenergetic change resulted in a growth disadvantage of Kras ${ }^{G 12 D}$-driven as well as Kras wild-type lung cancer cells. Cell-autonomous re-expression of both wild-type and mutant AIF (displaying an intact mitochondrial, but abrogated apoptotic function) in Aif-knockout $\mathrm{Kras}^{G 12 D}$ mice restored OXPHOS and reduced animal survival to the same level as AIF wild-type mice. In patients with non-small cell lung cancer, high AIF expression was associated with poor prognosis. These data show that AIF-regulated mitochondrial respiration and OXPHOS drive the progression of lung cancer.

Cell Research (2019) 29:579-591; https://doi.org/10.1038/s41422-019-0181-4

\section{INTRODUCTION}

AIF (apoptosis-inducing factor) was first cloned as a caspaseindependent death effector released from mitochondria. ${ }^{1}$ Since then, multiple publications pointed to an important, albeit not essential, function of AIF in several cell death scenarios in multiple species including mouse, $S$. cerevisiae, C. elegans, and D. melanogaster. ${ }^{2-4}$ Importantly, similar to the key cell death effector molecule cytochrome c, AIF not only contributes to cell death pathways, but also exerts a vital housekeeping function inside mitochondria, where it determines the rate of oxidative phosphorylation (OXPHOS) via posttranscriptional regulation of complex I proteins in the mitochondrial respiratory chain. ${ }^{3,5-8}$ Mice with tissue-specific Aif deletions or a hypomorph Aif mutation exhibit organ-specific complex I deficiency and enhanced glycolysis, confirming a key function for AIF in mitochondrial respiration. ${ }^{3,5-8}$ Since AIF is a protein with a dual function in cell death and OXPHOS, we tested whether genetic modulation of Aif would affect lung cancer tumorigenesis.

Cancer cells have a fundamentally different metabolic profile from that of normal tissue and this shift away from mitochondrial ATP synthesis via OXPHOS towards a high rate of glycolysis has long been recognized as a hallmark of cancer cells. ${ }^{9,10}$ This

\footnotetext{
${ }^{1}$ Department of Thoracic Surgery, Nanfang Hospital, Southern Medical University, Guangzhou, Guangdong, China; ${ }^{2} \mathrm{IMBA}$, Institute of Molecular Biotechnology of the Austrian Academy of Sciences, 1030 Vienna, Austria; ${ }^{3}$ Equipe 11 labellisée Ligue contre le Cancer, Centre de Recherche des Cordeliers, 75006 Paris, France; ${ }^{4}$ INSERM, U1138, 75006 Paris, France; ${ }^{5}$ Université Paris Descartes, Sorbonne Paris Cité, Paris, France; ${ }^{6}$ Metabolomics and Cell Biology Platforms, Gustave Roussy Cancer Campus, 94805 Villejuif, France;

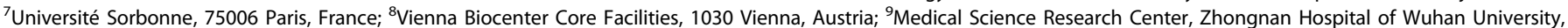
Wuhan, Hubei, China; ${ }^{10}$ Department of Neuronal Control of Metabolism, Max Planck Institute for Metabolism Research, Cologne, Germany; ${ }^{11}$ Research Center for Molecular Medicine of the Austrian Academy of Sciences, Vienna, Austria; ${ }^{12}$ Lunenfeld-Tanenbaum Research Institute, Mount Sinai Hospital, 600 University Avenue, Toronto, Canada; ${ }^{13}$ Vascular Cell Biology Unit, Department for Molecular Biomedical Research, VIB, Ghent, Belgium; ${ }^{14}$ Department of Biomedical Molecular Biology, Ghent University, Ghent, Belgium; ${ }^{15}$ Department of Pharmacology and Therapeutics, Rady Faculty of Health Sciences, University of Manitoba, Winnipeg, Canada; ${ }^{16}$ Gustave Roussy Cancer Campus, Villejuif, France; ${ }^{17}$ Faculty of Medicine, Université Paris-Saclay, Kremlin-Bicêtre, France; ${ }^{18}$ INSERM, U1030 Villejuif, France; ${ }^{19}$ Department of Cardiology, Renmin Hospital of Wuhan University, Wuhan, Hubei, China; ${ }^{20}$ Institute for Medical Informatics, Statistics and Documentation, Medical University Graz, Graz, Austria; ${ }^{21}$ Center for Diagnostics and Research in Molecular Biomedicine, Pathology Institute for Diagnostics and Research, Medical University Graz, Graz, Austria; ${ }^{22}$ Department of Experimental Pathology and Pathology of Laboratory Animals, Medical University Vienna and University of Veterinary Medicine Vienna, Vienna, Austria; ${ }^{23}$ Ludwig Boltzmann Institute for Cancer Research (LBI-CR), Vienna, Austria; ${ }^{24}$ INSERM, UMR1141, Hopital Robert Debre 48 Boulevard Serurier, 75019 Paris, France; ${ }^{25}$ Pôle de Biologie, Hôpital Européen Georges Pompidou, AP-HP, Paris, France; ${ }^{26}$ Suzhou Institute for Systems Biology, Chinese Academy of Sciences, Suzhou, Jiangsu, China; ${ }^{27}$ Department of Women's and Children's Health, Karolinska Institute, Karolinska University Hospital, Stockholm, Sweden and ${ }^{28}$ Department of Medical Genetics, Life Sciences Institute, University of British Columbia, Vancouver, Canada Correspondence: Guido Kroemer (kroemer@orange.fr) or Josef M. Penninger (josef.penninger@imba.oeaw.ac.at)

These authors contributed equally: Shuan Rao, Laura Mondragón
}

Received: 14 November 2018 Accepted: 5 May 2019

Published online: 27 May 2019 
glycolytic switch was termed the "Warburg effect", named after Otto Warburg who discovered this phenomenon in 1923 and later suggested it to constitute a fundamental cause of cancer. ${ }^{11}$ The Warburg effect has been proposed to support proliferation and the increased biosynthetic demands of cancer cells.

In this study, we use a genetic murine system to directly decrease the function of the respiratory chain (and hence to inhibit OXPHOS) in $\mathrm{Kras}^{\mathrm{G} 2 D^{2}}$-induced lung carcinogenesis by knocking out AIF. According to the Warburg hypothesis, this manipulation should accelerate oncogenesis. We indeed observed a bioenergetic Warburg shift in primary, non-transformed AIF mutant pneumocytes as well as at early stages of tumorigenesis. However, we observed that ablation of AIF reduced Kras ${ }^{G 12 D_{-}}$ driven lung carcinogenesis. Most importantly, cell-autonomous, engineered re-introduction of wild-type (WT) AIF or a mitochondria-confined mutant of AIF (that loses its extramitochondrial pro-death function) was sufficient to re-establish OXPHOS and Kras ${ }^{G 12 D}$-driven carcinogenesis.

To expand our findings, we analyzed both Kras mutant and WT human lung cancer cell lines from non-small cell lung cancer (NSCLC) patients. Interestingly, depletion of AIF resulted in impaired growth and clonogenic potential of all these human lung cancer cells, thereby confirming our conclusions with the genetic murine lung cancer data. Furthermore, by including lung cancer patients' data, we have demonstrated that both AIF mRNA and protein expression correlate with survival, and high levels of AIF are associated with poor prognosis. Surprisingly, by analyzing different NSCLC cohorts, we found that most genes encoding the complex I subunits of mitochondrial respiratory chain or their assembly factors were overexpressed in NSCLC tissues as compared to normal adjacent lung tissues. All these findings collectively confirm and strengthen the conclusion that
OXPHOS is supportive for lung cancer development in general, regardless of genetic background.

\section{RESULTS}

Reduced lung cancer in Aif-deficient Kras ${ }^{G 12 D}$ mice

To determine the role of AIF in lung cancer, we crossed Aif $f / f t$ mice $^{3}$ with the Lox-Stop-Lox-Kras ${ }^{G 12 D}$ strain. Lox-Stop-Lox-Kras ${ }^{G 12 D}$ mice develop non-small-cell lung carcinomas (NSCLCs) upon Cre deletion and induction of the mutant $\operatorname{Kras}^{G 12 D}$ allele in a stepwise process that leads from epithelial hyperplasia to benign adenomas and malignant adenocarcinomas. ${ }^{12,13}$ We achieved expression of $\mathrm{Kras}^{G 12 D}$ and simultaneous deletion of Aif following adenoviral delivery of Cre recombinase (AdCre) through inhalation using the Ad5-CMV-Cre or Ad5-mSPC-Cre virus (Supplementary information, Fig. S1a). The Aif gene is located on the X chromosome. Thus, male mice expressing oncogenic Kras ${ }^{G 12 D}$ born from heterozygous Aif $^{+/ f l}$ mothers develop tumors that are either knockout for Aif $\left(\right.$ Aif $\left.^{f / y} \mathrm{Kras}^{\mathrm{G}}{ }^{\mathrm{G} D}\right)$, or normally express AIF (Aif ${ }^{+/ y} \mathrm{Kras}^{\mathrm{G12D}}$ ) as littermate controls. Intriguingly, loss of AIF in the $\mathrm{Kras}^{G 12 D}$-driven lung cancer model resulted in significantly prolonged survival as compared to control Aif ${ }^{+/ y}$ Kras $^{G 12 D}$ littermates (Fig. 1a). Quantification of overall tumor burden revealed a significant decrease of the tumor areas in the lungs of iif $^{f / y} \mathrm{Kras}^{G 12 D}$ mice compared to Aif $^{+/ y} \mathrm{Kras}^{G 12 D}$ controls at all time-points analyzed (Fig. 1b, c). Aifdeficient lung cancers showed efficient deletion of Aif, as determined by qPCR of micro-dissected tumors and by immunohistochemistry to detect loss of AIF protein (Supplementary information, Fig. S1b, c). As expected from previous studies, ${ }^{13}$ the tumors arose from surfactant protein (SP-C) expressing type II alveolar pneumocytes (Supplementary information, Fig. S1d). AIF deletion, which is known to compromise the function of a

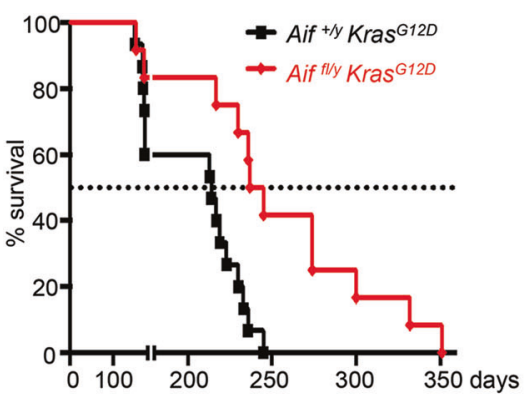

C

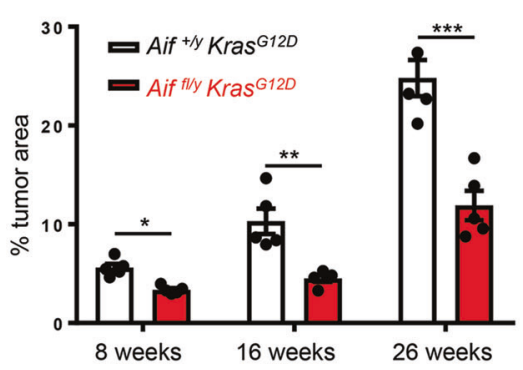

b

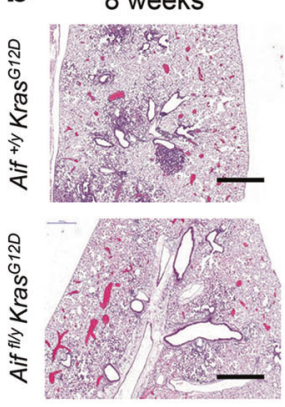

d

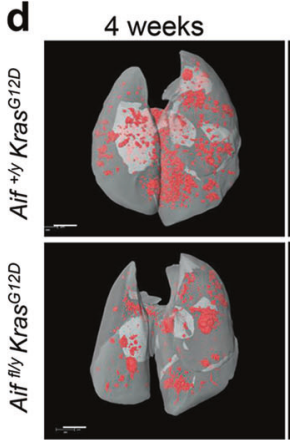

16 weeks

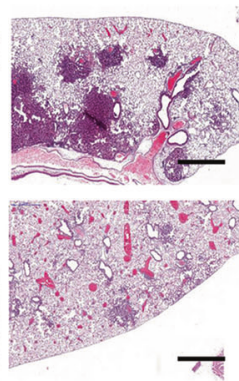

8 weeks

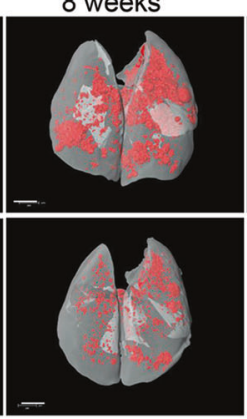

26 weeks

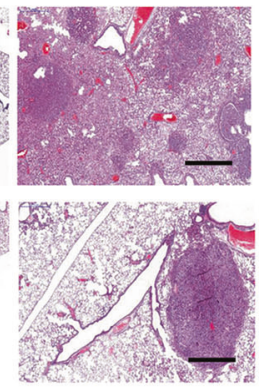

16 weeks

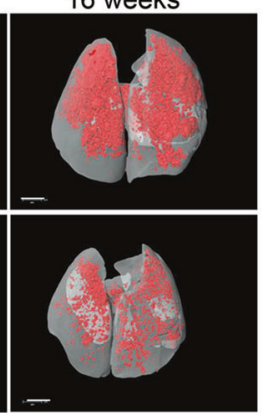

Fig. 1 Reduced lung cancer in Aif-deficient $\mathrm{Kras}^{\mathrm{G} 12 D}$ mice. a Aif deletion significantly prolongs the survival of mice infected with Ad5-CMV-Cre in comparison to their Aif WT controls. Kaplan-Meier plot. $P=0.0022$ (log rank test) for Aiff/y $\operatorname{Kras}^{G 12 D}(n=12)$ versus Aif ${ }^{+/ y} \mathrm{Kras}^{G 12 D}$ ( $(n=15)$ littermates. b Representative lung tumor sections (H\&E staining) in Aif ${ }^{f / y}$ Kras $^{G 12 D}$ and Aif ${ }^{+/ y}$ Kras $^{G 12 D}$ littermates at the indicated time points after Ad5-CMV-Cre inhalation. Scale bar, $2 \mathrm{~mm}$. c Quantification of overall tumor burden. Total tumor areas comprising hyperplasia, adenomas, and adenocarcinomas, were scored automatically by a Definiens software algorithm. Three planes from each lung were stained with $\mathrm{H} \& \mathrm{E}$ and analyzed in a blinded fashion. Data are shown as means \pm SEM. ${ }^{*} P<0.05 ;{ }^{* *} P<0.01 ;{ }^{* * *} P<0.001$ (Student's $t$-test). $n=5$ per genotype for each time point. d Micro-CT analysis of lung tumors of Aif ${ }^{f / y} \mathrm{Kras}^{G 12 D}$ and Aif ${ }^{+/ y} \mathrm{Kras}^{\mathrm{G} 12 \mathrm{D}}$ littermate mice, analyzed at the indicated weeks after Ad5CMV-Cre inhalation. Representative data from individual mice are shown. Scale bar, $2 \mathrm{~mm}$ 

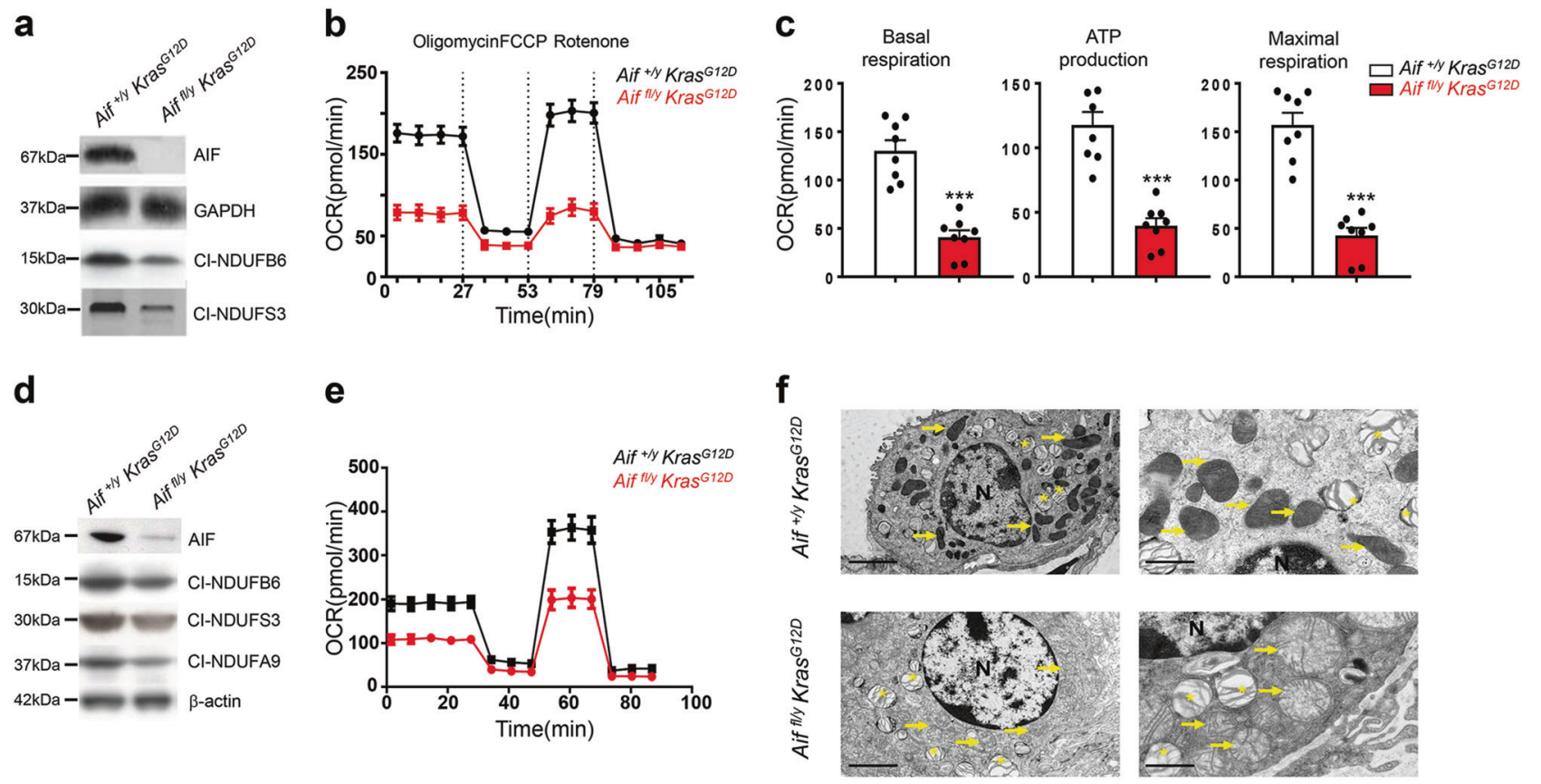

Fig. 2 Loss of AIF compromises OXPHOS and impairs mitochondrial structure in lung tumor cells. a Western blotting for AIF protein and the indicated OXPHOS complex I proteins in primary pneumocytes isolated from Aif $f^{7 / y} \mathrm{Kras}^{G 12 D}$ and Aif $^{+/ y} \mathrm{Kras}^{G 12 D}$ mice and consequently transfected with Ad5-CMV-Cre-eGFP in vitro. GAPDH was used as a loading control. b, c Representative OCR (b) and comparison (means \pm SEM) of basal respiration, ATP production and maximal respiration (c) in primary pneumocytes isolated from Aif ${ }^{f / y} \mathrm{Kras}^{G 12 D}$ and $\mathrm{Aif}^{+/ y} \mathrm{Kras}^{G 12 D}$ mice and consequently transfected with Ad5-CMV-Cre in vitro d Western blotting for AlF protein and the indicated OXPHOS complex I proteins in pneumocytes isolated from Aiff/y $\mathrm{Kras}^{G 12 D}$ and Aif ${ }^{+/ y} \mathrm{Kras}^{\mathrm{G} 2 D}$ mice 6 weeks after Ad5-CMV-Cre inhalation. $\beta$-actin was used as a loading control. e Representative OCR analysis of purified transformed pneumocytes isolated from Aif ${ }^{f / y} \mathrm{Kras}^{G 12 D}$ and $\mathrm{Aif}^{+/ y} \mathrm{Kras}^{G 12 D}$ mice 6 weeks after Ad5-CMV-Cre inhalation. f Representative electron microscopy images for tumor tissues isolated from Aif ${ }^{+/ y}$ Kras $^{G 12 D}$ (upper panels) and Aif ${ }^{A / y} K_{\text {ras }}{ }^{G 12 D}$ mice (lower panels) 18 weeks after Ad5-CMV-Cre inhalation. Note normal mitochondrial morphology with mostly intact cristae in AlF-competent tumors in contrast to swollen mitochondria with notable cristolysis in Aif-deficient lung tumor tissues (yellow arrows). Asterisks indicate lamellar bodies (Corpuscula lamellariae), rare cell organelles containing surfactant lipoproteins characteristic for type II pneumocytes. $\mathrm{N}$ indicates nuclei. Scale bars, $5 \mu \mathrm{m}$ for left panel and $2 \mu \mathrm{m}$ for right panel

respiratory chain complex $\mathrm{I}^{5,8}$ did not affect the integrity of respiratory chain supercomplexes, as analyzed by native gel electrophoresis (Supplementary information, Fig. S1e). These results show that genetic inactivation of Aif results in markedly reduced $\mathrm{Kras}^{\mathrm{G12D}}$-driven lung tumorigenesis.

We next assessed tumor initiation and staged the malignant progression of the lung cancers. Four weeks after Ad5-CMV-Cre inhalation, micro-CT already revealed markedly reduced tumor foci in $\mathrm{Aif}^{f / y} \mathrm{Kras}^{G 12 D}$ mice, and this reduction of tumor foci was observed throughout the entire observation period (Fig. 1d). Using established histopathological criteria ${ }^{14}$ at 4 weeks after Ad5CMV-Cre inhalation, Aif ${ }^{+/ y}$ Kras $^{\text {G12D }}$ control mice harbored multiple hyperplastic lesions and even small solid adenomas, whereas at this time point only small hyperplastic regions were observed in Aif $^{f / y}$ Kras $^{G 12 D}$ mice (Supplementary information, Fig. S2a). Eight weeks after Ad5-CMV-Cre inhalation, we again observed significantly less hyperplastic regions as well as reduced numbers of adenomas in Aif ${ }^{f / y} \mathrm{Kras}^{G 12 D}$ mice as compared to Aif ${ }^{+/ y} \mathrm{Kras}^{G 12 D}$ littermates (Supplementary information, Fig. S2b). At 16 weeks after Ad5-CMV-Cre infection, inactivation of Aif resulted in markedly reduced progression to adenocarcinomas (Supplementary information, Fig. S2c). Due to the large size of lung adenocarcinomas at 26 weeks after Ad5-CMV-Cre inhalation, it was not possible to reliably count individual tumors at this time point. Proliferation, as detected by Ki67 staining, was significantly reduced in hyperplastic regions, as well as in early (4 weeks after Ad5-CMV-Cre inhalation) adenomas from Aif ${ }^{f / y} \mathrm{Kras}^{G 12 D}$ mice (Supplementary information, Fig. S2d-f). Moreover, we observed enhanced expression of the senescence marker promyelocytic leukemia protein (PML) (Supplementary information, Fig. S2g, h), the expression of which correlates inversely with the malignancy and proliferative index of tumors. ${ }^{15}$ We detected only a few apoptotic cells (that stained positively for cleaved caspase-3) in Kras $^{G 12 D}$-induced lung tumors from both Aifl/y $\mathrm{Kras}^{\mathrm{G} 12 D}$ and Aif ${ }^{+/ y}$ Kras $^{G 12 D}$ mice, confirming previous reports on the anti-apoptotic potential of oncogenic Kras ${ }^{G 12 D} \cdot{ }^{15}$ Importantly, we did not find significant differences in the percentages of apoptotic cells among the different cohorts at all time-points analyzed (data not shown). Thus, loss of AIF delays Kras ${ }^{G 12 D}$-driven lung tumor initiation and malignant progression to adenocarcinomas.

Loss of AIF compromises OXPHOS and impairs mitochondrial structure

As AIF affects the onset and early stages in tumor development, we isolated primary pneumocytes from Aif ${ }^{f / y} \mathrm{Kras}^{\mathrm{G}}{ }^{\mathrm{G} D}$ and $\mathrm{Aif}^{+/ y} \mathrm{Kras}^{\mathrm{G}}{ }^{2 D}$ littermates and subsequently infected the cultured cells with Ad5CMV-Cre-eGFP to activate $\mathrm{Kras}^{\mathrm{G}}{ }^{2 D}$ expression and concomitantly delete Aif (Fig. 2a; Supplementary information, Fig. S3a). Deletion of AIF, as expected from previous studies, ${ }^{6,8}$ also resulted in a consequent reduction of proteins in the mitochondrial complex I (Fig. 2a). Aif mutant primary pneumocytes were then analyzed for their oxygen consumption rate $(\mathrm{OCR})^{16}$ to assess mitochondrial respiration. Aif ${ }^{f / y} \mathrm{Kras}^{G 12 D}$ cells consumed oxygen at a significantly lower basal level, produced much less ATP as well as exhibited a limited OCR increase in response to the uncoupler carbonylcyanide-p trifluoromethoxyphenylhydrazone (FCCP), yielding a reduced maximal respiratory capacity (as an indicator of the level of OXPHOS) (Fig. 2b, c). In addition, Aif deficiency caused a decrease in mitochondrial spare respiration capacity with a simultaneous reduction of proton leakage (Supplementary information, Fig. S3b). 
a
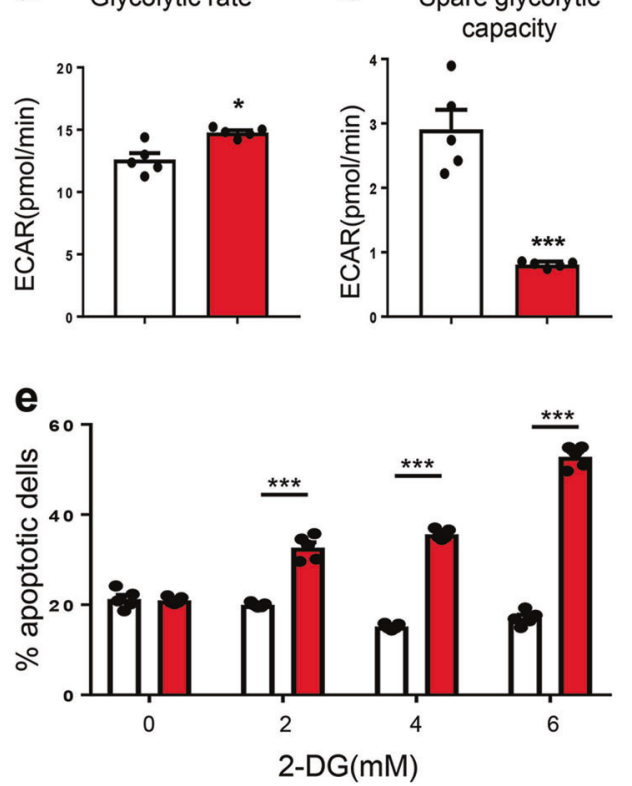

C

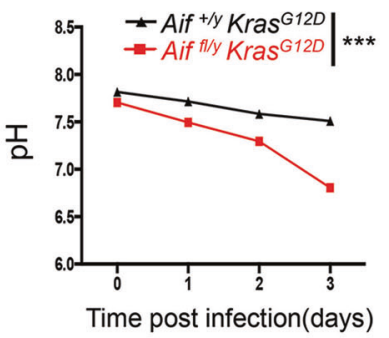

f

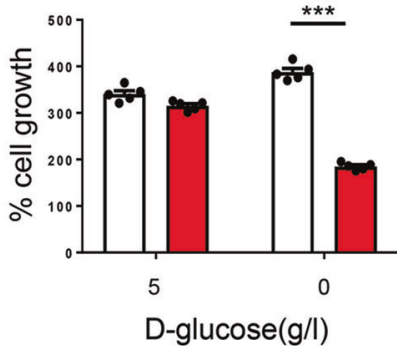

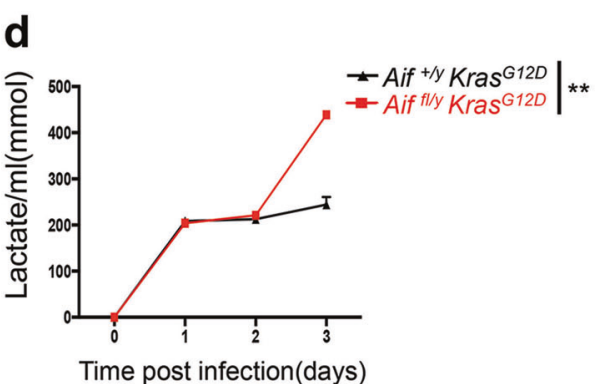

9 Aif Aif $^{+/ y} \mathrm{Kras}^{G 12 D} \mathrm{Kras}^{\mathrm{G12D}}$

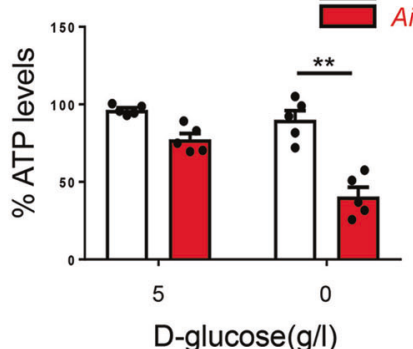

Fig. 3 AIF deficiency enhances glycolysis and sensitivity to glucose deprivation. a, b ECAR in pneumocytes isolated from Aif ${ }^{+/ y}$ Kras $^{G 12 D}$ and Aiff/y $\mathrm{Kras}^{\mathrm{G} 12 \mathrm{D}}$ mice 6 weeks after Ad5-CMV-Cre inhalation. Basal glycolytic rate after stimulation with glucose (a) and change in ECAR over baseline after oligomycin treatment (b). ${ }^{*} P<0.05 ;{ }^{* *} P<0.001$ (Unpaired two-sided $t$-test). $\mathbf{c}$, $\mathbf{d}$ pH measurements (c) and lactate production (d) in the culture media of primary pneumocytes isolated from Aif ${ }^{+/ y} \mathrm{Kras}^{G 12 D}$ and Aif ${ }^{f / y} \mathrm{Kras}^{G 12 D}$ mice 6 weeks after Ad5-CMV-Cre inhalation. Cells were seeded on day 0 with a density of $2.5 \times 10^{5}$ cells/well in a 6 -well plate. Data are shown as means \pm SEM. $n=5$ per genotype. ${ }^{* *} P<0.01$; ${ }^{* * *} P<0.001$ (Two-way ANOVA test). e Aif ${ }^{+/ y} \mathrm{Kras}^{G 12 D}$ and Aif ${ }^{A / y} \mathrm{Kras}^{G 12 D}$ pneumocytes were cultured for $72 \mathrm{~h}$ in the presence of the indicated concentrations of 2-DG followed by staining with PI to determine the frequency of dead cells. ${ }^{* * *} P<0.001$ (two-way ANOVA, Bonferroni's post hoc test). $\mathbf{f} \mathrm{Aif}^{+/ y} \mathrm{Kras}^{G 12 D}$ and $\mathrm{Aif}^{\mathrm{Aly}} \mathrm{Kras}^{\mathrm{G} 12 D}$ pneumocyte growth in the absence of glucose. Cells were cultured for three days in the presence $(5 \mathrm{~g} / \mathrm{L})$ or absence of glucose and their viability was determined. g Reduced ATP production in Aiff/y Kras ${ }^{G 12 D}$ cells upon glucose withdrawal. Pneumocytes were cultured for $36 \mathrm{~h}$ in the absence or presence of glucose and intracellular ATP levels were determined among the viable cell fractions. ATP content was normalized to the protein concentration of the samples. Data are shown as means \pm SEM. $n=5$ per genotype. ${ }^{*} P<0.05 ;{ }^{* *} P<0.01 ;{ }^{* * *} P<0.001$ (two-way ANOVA, Bonferroni's post hoc test)

We next isolated primary transformed pneumocytes from Aif $^{f / y} \operatorname{Kras}^{G 12 D}$ and Aif ${ }^{+/ y} \operatorname{Kras}^{G 12 D}$ littermates 6 weeks after in vivo Ad5-CMV-Cre infection. Of note, the mice were infected with 5 times higher viral titer than the normal concentration $\left(2.5 \times 10^{7} \mathrm{PFU}\right)$ to ensure that the majority of pneumocytes were infected (Fig. 2d). Similar to our short-term cultures, in vivo Ad5CMV-Cre treatment resulted in reduced basal respiration, ATP production, maximal respiration, spare respiratory capacity as well as proton leakage (Fig. 2e; Supplementary information, Fig. S3c). We also characterized mitochondria in freshly dissected tumor tissue from $\mathrm{Aif}^{f / y} \mathrm{Kras}^{G 12 D}$ and $\mathrm{Aif}^{+/ y} \mathrm{Kras}^{\mathrm{G12D}}$ mice 16 weeks after Ad5-CMV-Cre inhalation. No difference was found in mitochondria numbers between AIF-competent and -deficient tumor cells; however, Aiffly Kras ${ }^{G 12 D}$ tumor cells exhibited swollen mitochondria with dramatically decreased and disordered cristae structures (Fig. 2f). Thus, loss of AIF results in not only a general impairment of OXPHOS, but also an abnormal mitochondrial morphology.

Aif deficiency enhances glycolysis and sensitivity to glucose deprivation

Upon stimulation of glycolysis by addition of glucose, the basal extracellular acidification rate (ECAR, a surrogate of lactate release), was higher in Aif $^{f / y} \mathrm{Kras}^{G 12 D}$ pneumocytes as compared to Aif $^{+/ y} \mathrm{Kras}^{G 12 D}$ control cells (Fig. 3a). After addition of oligomycin, which suppresses ATP production by OXPHOS, the spare glycolytic activity increased in both Aif $^{f / y} \mathrm{Kras}^{\mathrm{G} 12 \mathrm{D}}$ and Aif $^{+/ y} \mathrm{Kras}^{\mathrm{G12D}}$ cells, albeit significantly more in Aif $^{+/ y} \mathrm{Kras}^{\mathrm{G} 12 D}$ pneumocytes (Fig. 3b), indicating that glycolysis in $A_{i f}{ }^{f / y} \mathrm{Kras}^{G 12 D}$ cells is occurring at a close-to-maximum rate that is barely increased when ATP synthesis from oxidative metabolism is inhibited. Increased glycolysis was also observed in ex vivo
Ad5-mSPC-Cre-treated Aif ${ }^{f / y}$ Kras $^{G 12 D}$ pneumocytes, as determined by measuring extracellular $\mathrm{pH}$ and the release of lactate into the culture media over several days or by quantifying ECAR in shortterm experiments (Fig. 3c, d). An inhibitor of glycolysis, 2-Ddeoxyglucose (2-DG), was next utilized to determine whether the survival of $A i f^{f / y} \mathrm{Kras}^{G 12 D}$ pneumocytes depends on glycolysis. Control $\mathrm{Aif}^{+/ y} \mathrm{Kras}^{\mathrm{G} 12 \mathrm{D}}$ cells were not affected by 72 -h incubation with 2-DG, even at a concentration of $6 \mathrm{mM}$, whereas Aif $f^{f / y}$ Kras $^{G 12 D}$ cells exhibited a dose-dependent increase in mortality in response to 2-DG (Fig. 3e). Similarly, glucose withdrawal had no apparent effect on Aif ${ }^{+/ y}$ Kras $^{G 12 D}$ cells, yet compromised the growth of Aifl/y $\mathrm{Kras}^{G 12 D}$ cells (Fig. 3f). Of note, upon culturing in the presence of glucose, the cell numbers and rates of cell death (determined by propidium iodide (PI) staining) of both Aif $^{+/ y} \mathrm{Kras}^{G 12 D}$ and Aif ${ }^{7 / y} \mathrm{Kras}^{G 12 D}$ pneumocytes were comparable (data not shown). Finally, we observed a marked decline in intracellular ATP levels in Aif ${ }^{f / y}$ Kras $^{G 12 D}$ pneumocytes cultured in glucose-depleted conditions (Fig. $3 \mathrm{~g}$ ). These results indicate that Aif deletion leads to OXPHOS deficiency and a switch in cellular metabolism towards glycolysis. In Aiffly $\mathrm{Kras}^{G 12 D}$ pneumocytes, OXPHOS marginally contributes to the synthesis of ATP, which is mostly provided by glycolysis, contrasting with Aif $^{+/ y} \mathrm{Kras}^{\mathrm{G} 12 \mathrm{D}}$ cells, in which most of the ATP is generated by OXPHOS.

Stable Aif depletion impairs clonogenic potential and proliferation of both KRAS WT and KRAS mutant human lung cancer cells Stable knockdown of AIF in KRAS-mutant human NSCLC A549 cells compromised the expression of CHCHD4, a mitochondrial AIF interactor that is important for respiratory chain biogenesis, ${ }^{8}$ resulting in the reduction of proteins from the respiratory chain such as CIII-UQCRC2, CIV-COXII, and CI-NDUFB8 (Fig. 4a; 
a

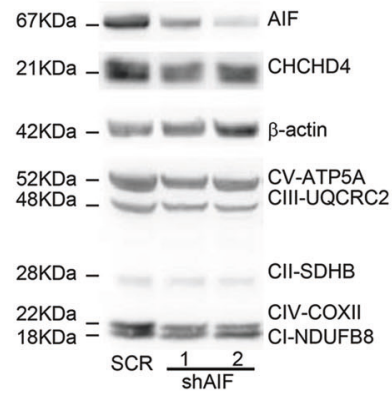

d

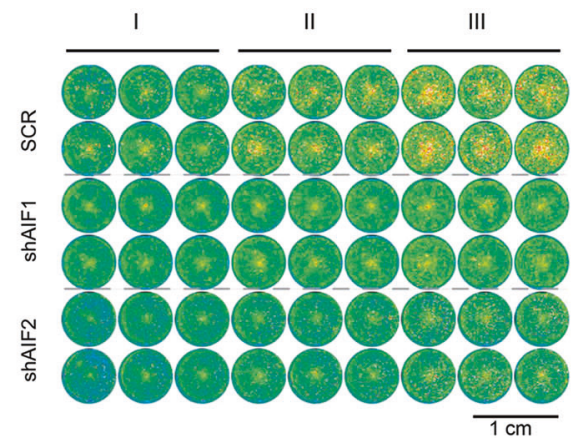

C

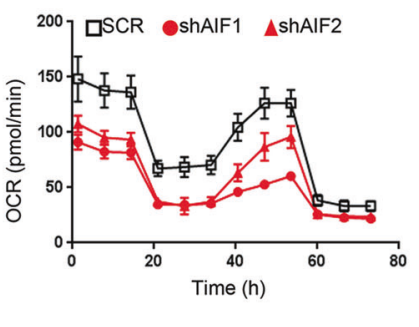

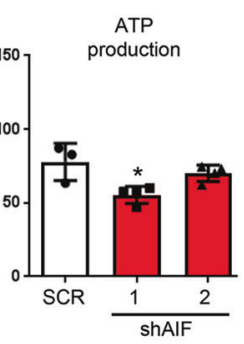

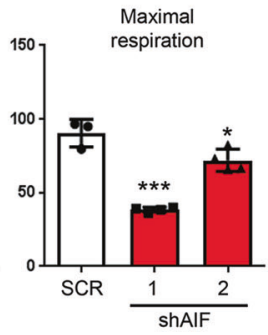

\section{1}

e

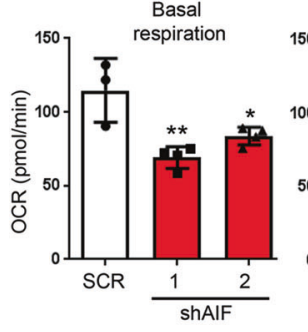

$$
\text { e }
$$
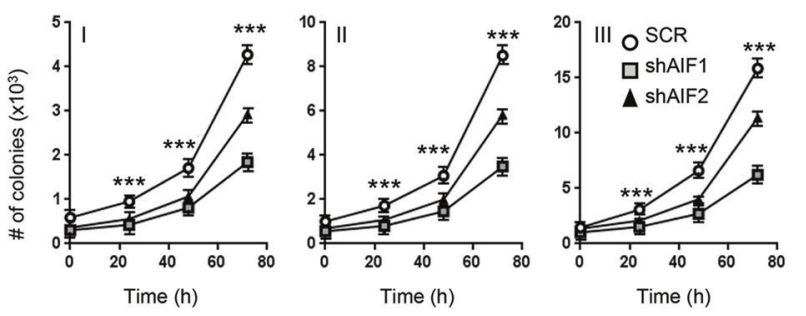

Fig. 4 AIF knockdown results in suppression of OXPHOS, clonogenic potential and cell proliferation in human NSCLC A549 lung tumor cells. a Cellular extracts from A549 clones, generated by lentiviral transduction with shRNA scramble (SCR) or two different shRNA constructs targeting AIF (shAIF1 and ShAIF2), were analyzed by immunoblot for the abundance of the indicated proteins. See Supplementary information, Fig. S6 for quantification. b Representative OCR of A549 SCR, shAIF1 and shAIF2 clones under basal conditions or following the addition of $1 \mu \mathrm{M}$ oligomycin, $1.5 \mu \mathrm{M}$ of the uncoupler FCCP or $0.5 \mu \mathrm{M}$ of the electron transport inhibitor rotenone $(n=5)$. c Quantification of basal respiration, ATP consumption and maximal respiration levels for SCR, shAIF1, and shAIF2 A549 clones. Results were normalized versus a SCR clone cells/well number and expressed as means \pm SEM (experiment was done in triplicate with similar results). d Representative cell growth assay of SCR, shAIF1 and shAIF2 A549 lung tumor clones (I, 500 cells/well; II, 1000 cells/well, and III, 2000 cells/well), analyzed by GFP fluorescence at $72 \mathrm{~h}$ post-seeding. e The indicated SCR, shAIF1 and shAIF2 A549 clones were plated (I, 500 cells/well; II, 1000 cells/well and III, 2000 cells/well) and colony numbers were quantified by GFP fluorescence at 0, 24, 48 and $72 \mathrm{~h}$ post-seeding. Values are means \pm SEM of a representative experiment containing 24 repeats of each condition (experiment was done in triplicate with similar results). Unpaired twosided $t$-test, ${ }^{*} P<0.05 ;{ }^{* *} P<0.01 ;{ }^{* *} P<0.001$ in case of immunoblot and oxygen consumption studies and two-way ANOVA and Bonferroni's post hoc test in case of cell proliferation studies, compared to control SCR cells

Supplementary information, Fig. S4), OXPHOS, ATP generation (Fig. 4b, c), clonogenic potential, as well as proliferation (Fig. 4d, e). This effect was phenocopied by knockdown of CHCHD4 in A549 cells that also compromised the expression of respiratory chain components (but not that of AIF) and resulted in reduced proliferation and diminished clonogenic potential of these human lung cancer cells (Supplementary information, Fig. S5a-d). Transfection-enforced overexpression of a CHCHD4 variant (CHCHD4exo) that directly incorporates into mitochondria restored the clonogenic potential of AIF-depleted A549 cells (data not shown). Using the same strategy, we further analyzed whether knocking down Aif by means of shRNA would affect the clonogenic potential and/or proliferation of additional human lung cancer cell lines, including five different KRAS-mutated cell lines (H460, H727, A427, H1650, and H358), and two lung cancer lines that are WT for KRAS (H1437 and H1975). Aif knockdown significantly impaired the clonogenic capacity of H1437, H727, A427, H1650, H358, and also above-shown A549 cells (Fig. 5a-d). Moreover, individual clones originating from AIF-depleted $\mathrm{H} 1437$, $\mathrm{H} 1975, \mathrm{~A} 549, \mathrm{H} 460$, and $\mathrm{H} 727$ cells exhibited reduced proliferation compared to their AIF-competent controls (Supplementary information, Fig. S6a-e). Importantly, none single colony could be obtained from A427, H1650, and H358 cells after AIF knockdown, indicating that AIF is essential for the proliferation of these human lung cancer cell lines. Altogether, these findings indicate that AIF-sustained OXPHOS may be essential for the optimal proliferation of both Kras-mutated and Kras WT human lung cancer cells.
Re-expressing WT or mitochondria-anchored AIF restores lung cancer sensitivity

So far, we have shown that loss of AIF markedly delays the onset and progression of oncogenic Kras ${ }^{G 12 D}$-driven lung cancer and that Aif-deficient pneumocytes exhibit a Warburg-like bioenergetic profile. However, AIF has a dual role, depending on its subcellular localization: (i) as a positive regulator of OXPHOS in mitochondria, and (ii) as a caspase-independent death effector when released from mitochondria, a process that requires permeabilization of the mitochondrial outer membrane as well as proteolytic cleavage of the AIF protein. ${ }^{17}$ To distinguish between these two roles, we constructed two different knock-in (ki) mouse lines, namely (i) WT ki mice that express a WT Aif transgene inserted into the ROSA26 locus $\left(R 26^{A I F-W T /+}\right)$, preceded by a Lox-Stop-Lox cassette, and (ii) MT ki mice that express mutant Aif $\Delta 96-110$ inserted into the same locus $\left(R 26^{\text {AlF-Mut/+ }}\right)$. This design ensures that both transgenes are only introduced once into the genome, do not cause collateral mutations, and are transcribed and regulated in a similar fashion (Fig. 6a, b). AlF $\Delta 96-110$ cannot be cleaved by cathepsins/calpains and thus is retained in mitochondria, precluding its translocation to the cytosol and the nucleus. ${ }^{17}$ Accordingly, in cell culture experiments, WT AIF translocates to the nucleus following a death stimulus, whereas AIF $\Delta 96-110$ remains in the mitochondria and hence cannot participate in lethal signaling pathways. ${ }^{17}$ Transgenic WT Aif (WT ki) and Aif $496-110$ (MT ki) were indistinguishable in their capacity to reverse the embryonic lethality that is observed in mice bearing the actin Cre-Aif $f^{f / y}$ genotype. ${ }^{18}$ In this setting (Fig. 6a, b), the endogenous Aif gene is universally deleted by Cre in all cell 
a
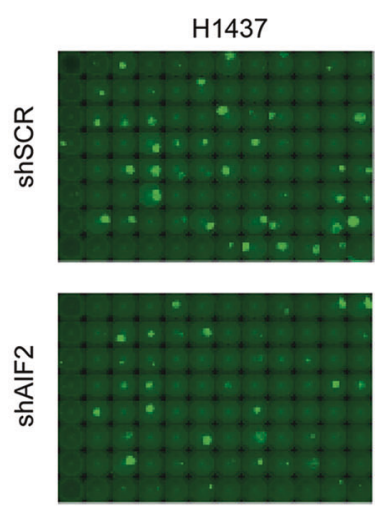

A427
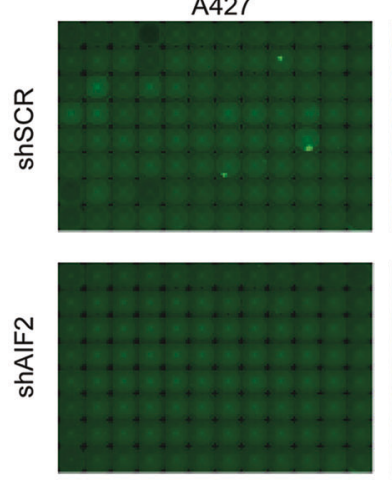
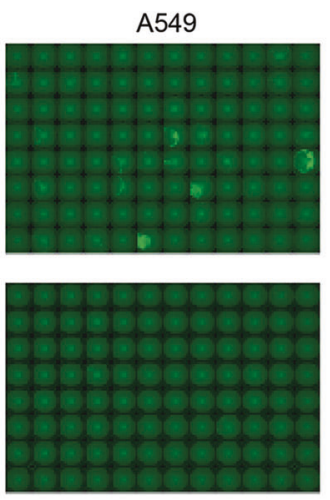

H358
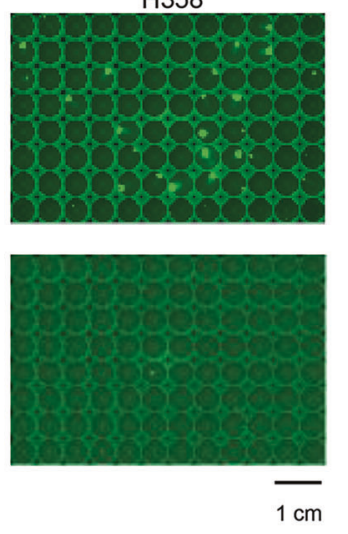
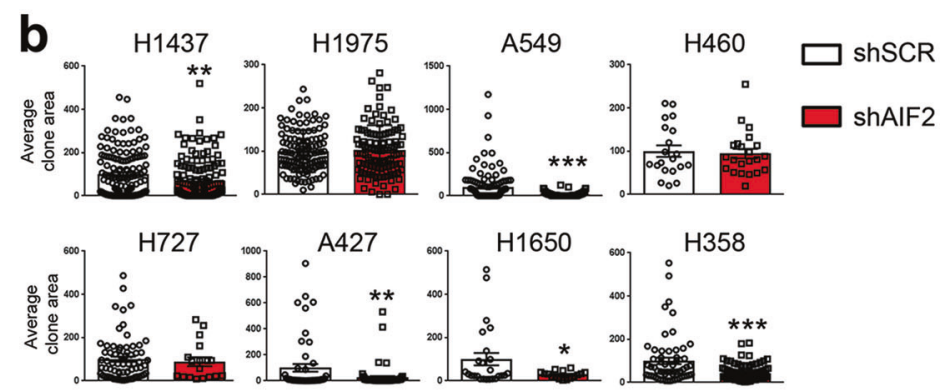

C
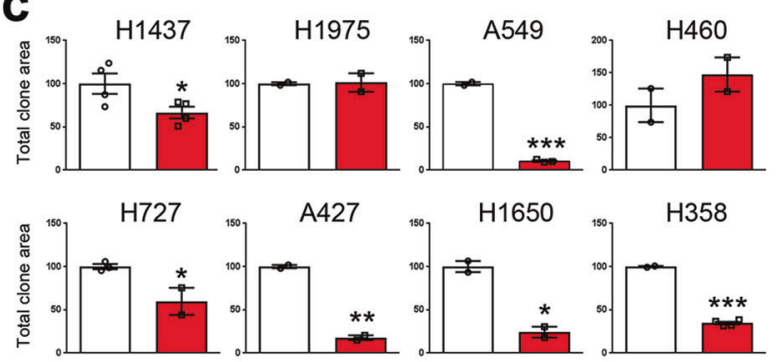

A427

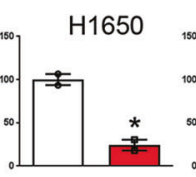

H358
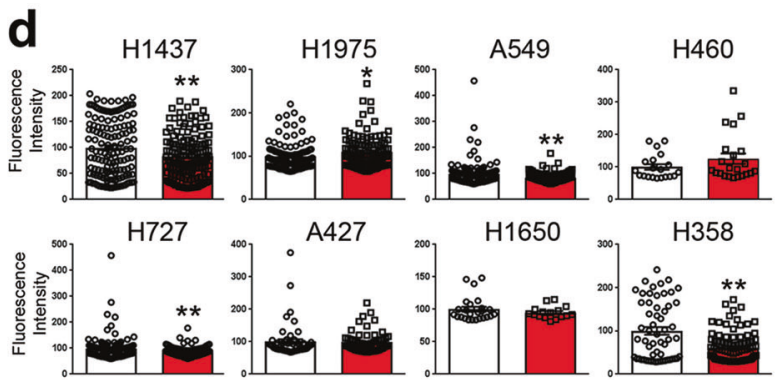

$\mathrm{H} 1650$

$\mathrm{H} 358$

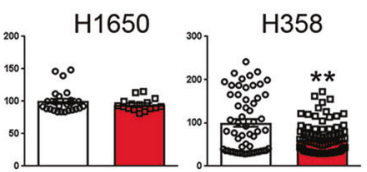

Fig. 5 Stable Aif depletion impairs colony formation of both KRAS WT and KRAS-mutated human lung cancer cells. KRAS WT cells (H1437 and $\mathrm{H} 1975)$ and KRAS-mutated human lung cancer cell $(\mathrm{H} 460, \mathrm{H} 727, \mathrm{~A} 427, \mathrm{H} 1650$, and $\mathrm{H} 358)$ were transduced with lentiviral vectors expressing shAIF-GFP (or shSCR-GFP as a control), and GFP-positive clones were selected by cytofluorometric sorting. A549 cells were used as an internal control. a Representative images of different 96-well plates seeded with one GFP-positive cell per well generated from different cell lines. The images were acquired after 7-12 day-long cell culture. b-d Quantification of average GFP ${ }^{+}$clone area (b), total GFP $^{+}$clone area (c), average fluorescence intensity (d). The values obtained for the control shSCR were set as 100, and the values of shAIF were normalized to individual shSCR controls. Results are expressed as means \pm SEM of a representative experiment (experiments were done in triplicates). ${ }^{*} P<0.05 ;{ }^{* *} P<0.01 ;{ }^{* * *} P<0.001$ (Student's $t$-test)

types yet replaced by transgene-encoded AIF following the Cremediated removal of the Lox-Stop-Lox cassette in the $5^{\prime}$ untranslated region (5' UTR) of the transgenes. Mice expressing transgenic WT Aif or Aif $\Delta 96-110$ in lieu of endogenous AIF similarly displayed normal, healthy appearance (data not shown), indicating that the knocked-in WT as well as mitochondrial-anchored mutant AIF $\Delta 96-$ 110 can definitely rescue the lethality of Aif global knockout mice, i.e., both Aif transgenes are functional in vivo. These data also indicate that the extramitochondrial function of AIF is dispensable for its contribution to normal development.

In the next step, we introduced the Aif (WT ki) and Aif $\Delta 96-110$ (MT ki) transgenes into an $A i^{f / y} \mathrm{Kras}^{G 12 D}$ background. In the resulting mouse lines, infection with Ad5-CMV-Cre resulted in Kras ${ }^{G 12 D}$ induction, deletion of the endogenous Aif gene, and the additional deletion of the Lox-Stop-Lox cassette in the $5^{\prime}$ UTR of the knock-in Aif transgenes, thereby replacing endogenous AIF by either WT AIF or mitochondria-anchored mutant AIF (Supplementary information, Fig. S7a). As in the initial experimental cohorts, Aiff/y $\mathrm{Kras}^{G 12 D}$ mice survived significantly longer after Ad5-CMV-Cre inhalation than AIFexpressing control littermates (Fig. 6c). Re-expression of WT AIF in Aif-knockout mice resulted in significantly reduced survival to a level comparable to Aif ${ }^{+/ y} \mathrm{Kras}^{G 12 D}$ and Aif ${ }^{+/ y}$ WT ki Kras ${ }^{G 12 D}$ mice. Importantly, replacement of endogenous AIF by mitochondriaanchored mutant AIF also restored the cancer phenotype of $A i f^{f / y}$
Kras $^{G 12 D}$ mice (Fig. 6c). Control Aif $f^{+/ y}$ WT ki Kras ${ }^{G 12 D}$ and Aif ${ }^{+/ y}$ MT ki Kras $^{G 12 D}$ mice behaved as Aif ${ }^{+/ y}$ Kras $^{G 12 D}$ mice with respect to overall survival and lung carcinogenesis, indicating that the transgenes had no additive effect in mice that expressed endogenous AIF. Immunoblot analysis confirmed efficient reexpression of the WT as well as mitochondria-anchored AIF protein in purified tumor cells from both Aiffly WT ki Kras ${ }^{G 12 D}$ and Aiff/y MT ki Kras $^{G 12 D}$ mice (Supplementary information, Fig. S7b). Furthermore, immunofluorescence staining revealed that re-introduced WT AIF was able to translocate from mitochondria to nuclei upon death stimulation, while AIF $\Delta$ 96-110 (MT AIF) was retained in mitochondria (Supplementary information, Fig. S7c). In line with reduced survival, quantification of overall tumor burden showed that, at all time-points analyzed, re-expression of WT or mutant AIF resulted in increased lung tumor burden that was comparable to the Aif ${ }^{+/ y}$ Kras $^{G 12 D}$ cohorts (Fig. 6d, e; Supplementary information, Fig. S7d, e).

We next staged the malignant progression of lung tumors. Four weeks after Ad5-CMV-Cre infection, only few hyperplastic regions were visible in $A^{A f l / y} \mathrm{Kras}^{G 12 D}$ mice, whereas the Aif ${ }^{+/ y} \mathrm{Kras}^{G 12 D}$ control mice already exhibited multiple hyperplastic lesions and small adenomas (Supplementary information, Fig. S8a, b). Importantly, the Aif//y WT ki Kras ${ }^{G 12 D}$ and Aiffly MT ki Kras ${ }^{G 12 D}$ knock-in mice developed similar numbers of hyperplastic lesions and adenomas to the Aif $^{+/ y} \mathrm{Kras}^{\mathrm{G12D}}$ control mice (Supplementary 
a

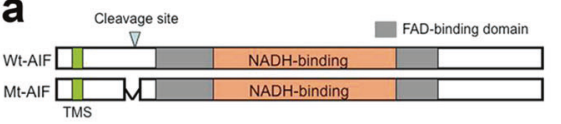

b

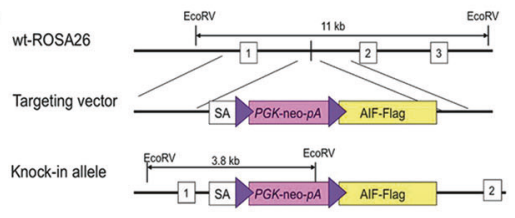

Cre-excised allele

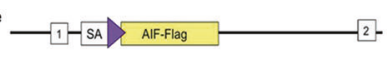

C

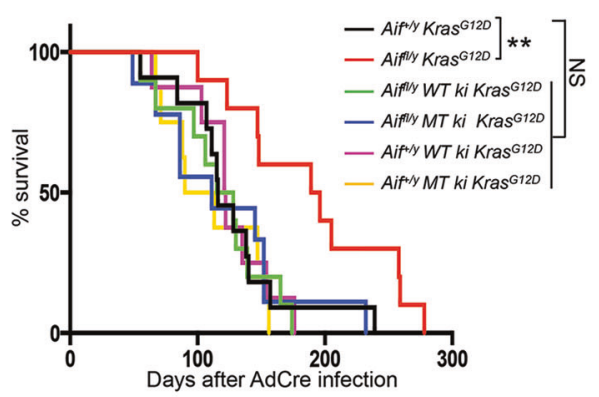

e

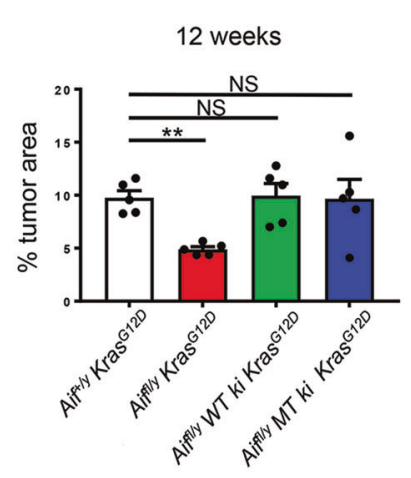

f

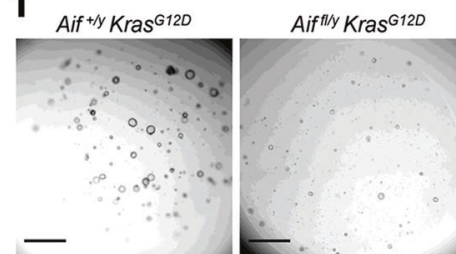

Aiffly WT ki Kras G12D Aiffly MT ki Kras ${ }^{G 12 D}$

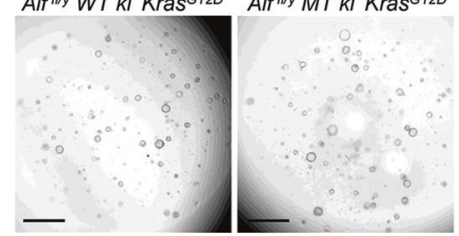

g

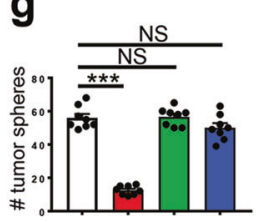

i

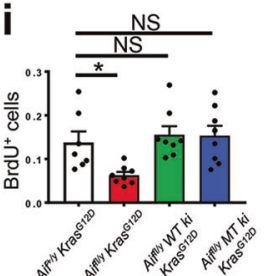

d

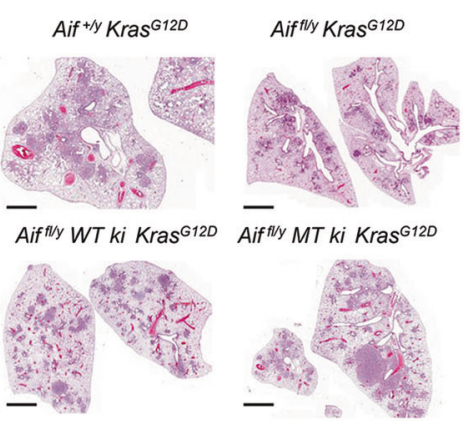

h

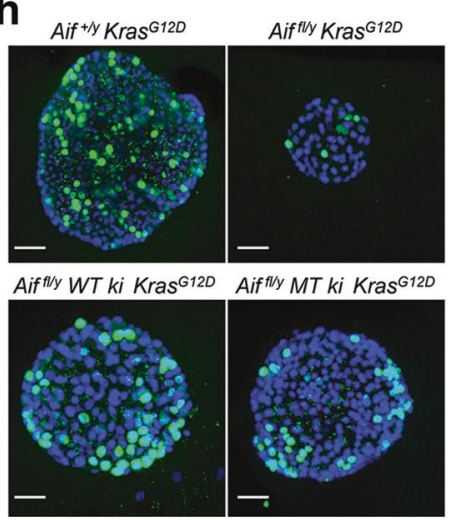

Fig. 6 Re-expressing WT or mitochondria-anchored AIF restores lung cancer sensitivity. a Schematic representation of the WT AIF (WT-Aif) and mitochondria-anchored mutant AIF $\Delta$ 96-110 (MT-Aif). TMS, transmembrane sequence. The cathepsin/calpain cleavage site is indicated. b Knock-in targeting strategy to insert WT-Aif and MT-Aif transgenes into the ROSA26 locus. Exons are shown as 1, 2, and 3. HSV-tk = herpes simplex promoter; PGK-neo-PA $=$ neomycin cassette for selection; AIF-FLAG $=$ AIF (WT or mutated) with a $3 \times F L A G$ tag, EcoR V $=$ restriction sites for Southern blotting. c Kaplan Meier survival plots for Aif ${ }^{+/ y} \operatorname{Kras}^{G 12 D}(n=11)$, Aif $^{f / y} \mathrm{Kras}^{G 12 D}(n=10), \operatorname{Aif}^{f / y}$ WT ki Kras ${ }^{G / 2 D}(n=10)$, Aif $^{f / y}$ MT ki Kras ${ }^{G 12 D}(n=9)$, Aif ${ }^{+\not y}$ WT ki Kras ${ }^{G 12 D}(n=9)$, and Aif ${ }^{+/ y} M T$ ki Kras ${ }^{G 12 D}(n=8)$ mice. ${ }^{* * P}<0.01$; NS, not significant (log rank test). d Representative lung sections (H\&E staining) of Aif $^{+/ y} \mathrm{Kras}^{G 12 D}$, Aifl/y Kras ${ }^{G 12 D}$, Aif ${ }^{7 / y}$ WT ki Kras ${ }^{G 12 D}$ and Aif ${ }^{f / y}$ MT ki Kras ${ }^{G 12 D}$ mice, analyzed at 12 weeks after Ad5-CMV-Cre infection. Scale bar, $2 \mathrm{~mm}$. e Quantification of overall tumor burden in the indicated cohorts analyzed 12 weeks after Ad5-CMV-Cre inhalation ( $n=5$ for each genotype). Three planes from each lung were scored automatically by an algorithm programmed and executed using the Definiens software suite program. Data are shown as means \pm SEM. ${ }^{* *} P<0.01 ;$ NS, not significant (twoway ANOVA analysis, Dunnett's multiple comparisons test). $\mathbf{f}$ Representative images of tumor spheroids derived from purified Aif ${ }^{+/ y} K$ ras $^{G 12 D}$ and $A i f^{f / y} K r a s^{G 12 D}$ primary lung tumor cells. Images were acquired 4 days after cells were seeded in Matrigel (5000 primary tumor cells per well). The experiment was designed with 6 replicates for each condition and repeated with 3 different mice for each group. Scale bar, 1 mm. g Quantitative analysis (means \pm SEM) of tumor spheroid numbers described in $\mathbf{f} .{ }^{* * *} P<0.001$; NS, not significant (Unpaired, two-sided $t$-test). h Representative images for BrdU staining of tumor spheroids derived from Aif ${ }^{+/ y} \mathrm{Kras}^{\mathrm{G} 12 D}$ and Aif ${ }^{f / y} \mathrm{Kras}^{\mathrm{G} 12 D}$ primary lung tumor cells seen as in f. BrdU labeling $(10 \mu \mathrm{M} / \mathrm{mL})$ was performed for $2 \mathrm{~h}$. Experiments were performed with 6 replicates for each condition and repeated with 3 different mice for each genotype. Sections were counter-stained with DAPI. i Quantifications (means \pm SEM) of BrdU ${ }^{+}$cells within tumor spheroids shown in $\mathbf{h} .{ }^{*} P<0.05$; NS, not significant (Unpaired, two-sided $t$-test)

information, Fig. S8a, b). At 8 weeks and 16 weeks after Ad5-CMVCre infection, we observed markedly less tumor burden, in particular, reduced numbers of adenocarcinomas in Aif $^{f / y} \mathrm{Kras}^{\mathrm{G}}{ }^{2 \mathrm{D}}$ as compared to Aif ${ }^{+/ y} \mathrm{Kras}^{G 12 D}$ mice; restoration of AIF expression with WT as well as mitochondria-anchored AIF resulted in increased adenomas and adenocarcinomas, phenocopying the control mice that express endogenous AIF (Supplementary information, Fig. S8b, c). In conclusion, re-expression of WT and mitochondria-anchored AIF similarly rescues the endogenous AIF deficiency and accelerates the onset and progression of Kras ${ }^{G 12 D_{-}}$ driven lung cancer.

To further investigate the functional relevance of AIF and AIFregulated OXPHOS in tumor cell proliferation as well as tumor stem-like properties, we employed a recently developed 3D tumor spheroid culture assay ${ }^{19}$ with purified primary pneumocytes isolated from Aif ${ }^{+/ y} \mathrm{Kras}^{G 12 D}$, Aif $^{f / y} \mathrm{Kras}^{\mathrm{G} 12 D}$, Aif $^{f / y}$ WT ki Kras ${ }^{G 12 D}$, Aiffly MT ki Kras ${ }^{G 12 D}$ mice 6 weeks after Ad5-mSPC-Cre infection. Following seeding the same numbers of tumor cells from each genotype, we detected a major decrease in the numbers of tumor spheroids derived from Aif $^{f l y} \operatorname{Kras}^{G 12 D}$ mice as compared to Aif $^{+/ y}$ Kras $^{G 12 D}$ mice (Fig. 6f, g). Re-expression of WT and mutant AIF could almost completely restore the stem-like property to form tumor spheroids to the level of the Aif ${ }^{+/ y} \mathrm{Kras}^{\mathrm{G} 12 \mathrm{D}}$ control (Fig. $6 \mathrm{f}, \mathrm{g}$ ). We next determined the proliferation capacity of these tumor spheroids originated from AIF-competent or -deficient tumor cells by BrdU labeling. Again, we observed significantly less BrdU-positive cells in Aiffly Kras ${ }^{\text {G12D }}$ tumor spheroids when compared to AIF WT, AIF WT knock-in and AIF mutant knock-in tumor spheroids (Fig. 6h, i). Addition of the OXPHOS inhibitor oligomycin efficiently blocked tumor spheroid formation at rather low concentrations (Supplementary information, Fig. S9a). Thus, AIF and AIF-regulated OXPHOS control lung cancer stem-like cell expansion and proliferation.

Restoration of WT or mitochondria-anchored AIF eliminates mitochondrial respiration defect

The re-introduction of either WT or mitochondria-anchored AIF into $A i^{f / y} \mathrm{Kras}^{G 12 D}$ pneumocytes could enhance their respiratory 
a

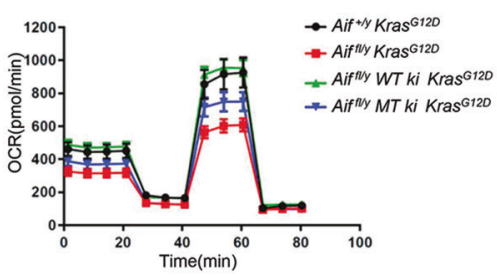

C

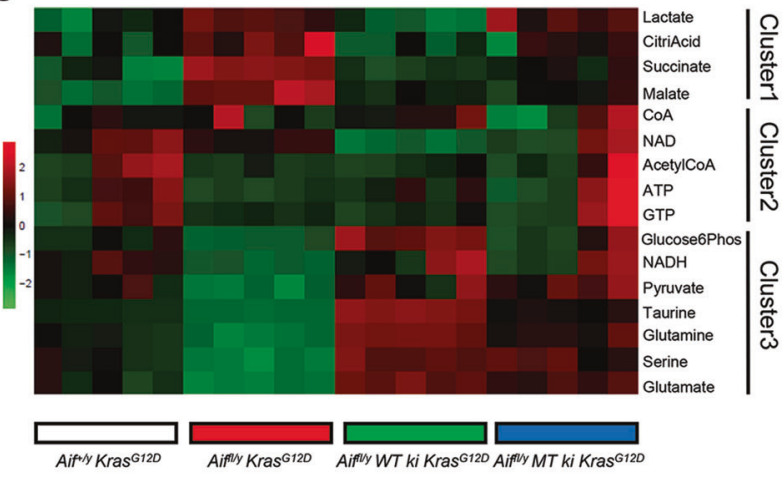

b

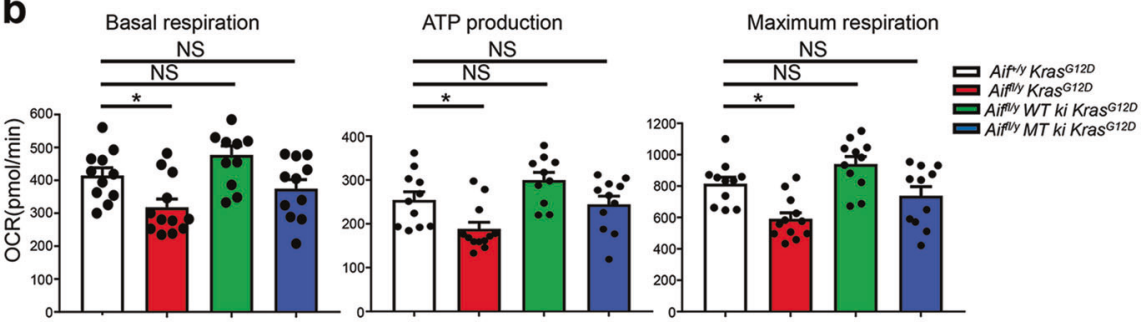

d

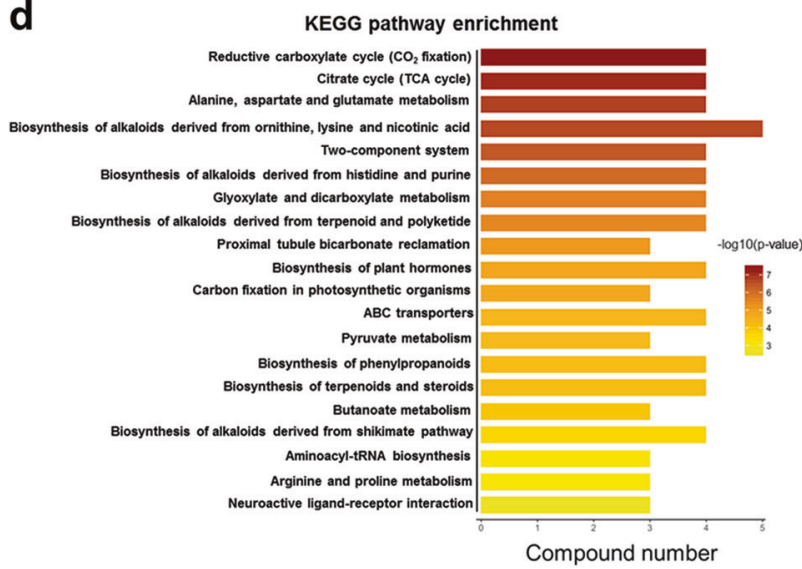

Fig. 7 Restoration of WT or mitochondria-anchored AIF eliminates mitochondrial respiration disadvantage. a, b Representative OCR (a) and comparison of basal respiration, ATP production and maximal respiration (b) in primary purified tumor cells derived from Aif ${ }^{+/ y}$ Kras $^{G 12 D}$ $\mathrm{Aif}^{f / y} \mathrm{Kras}^{G 12 D}$, Aiff/y WT ki Kras ${ }^{G 12 D}$, and Aiff/y MT ki Kras ${ }^{G 12 D}$ mice 6 weeks after Ad5-mSPC-Cre inhalation. Data are shown as means \pm SEM. ${ }^{*} P<0.05 ;{ }^{* *} P<0.01$; NS, not significant (Two-way ANOVA, Bonferroni's post hoc test). The experiment was designed with 12 replicates for each condition and repeated with 3 different mice for each genotype. c Determination of metabolite content from purified tumor cells derived from Aif ${ }^{+/ y} \mathrm{Kras}^{G 12 D}$, Aif/y $\mathrm{Kras}^{G 12 D}$. Aiff/y WT ki Kras ${ }^{G 12 D}$, and Aif ${ }^{f / y}$ MT ki Kras ${ }^{G 12 D}$ mice 8 weeks after Ad5-mSPC-Cre inhalation. Data are presented in a heatmap. Experiments were performed with 3 replicates for each condition and repeated with 5 different mice for each genotype. d KEGG pathway enrichment assay based on metabolite concentration obtained from Aif ${ }^{+/ y} \mathrm{Kras}^{\mathrm{G} 12 D}$, Aif $^{f / y} \mathrm{Kras}^{\mathrm{G} 12 D}$, Aif/y $\mathrm{WT}^{\mathrm{k} i}$ $\mathrm{Kras}^{G 12 D}$, and Aifl/y $M T$ ki Kras ${ }^{G 12 D}$ mice 3-4 weeks after Ad5-mSPC-Cre inhalation. Red box indicates the tricarboxylic acid (TCA) cycle, one of the top enriched pathways after AIF depletion

capacity to levels comparable to the Aif $^{+/ y}$ Kras $^{G 12 D}$ control (Fig. 7a, b; Supplementary information, Fig. S9b). To extend this characterization, we performed comparative targeted metabolomic analysis with purified tumor cells that were freshly isolated from Aif $f^{+/ y}$ Kras $^{G 12 D}$, Aif $^{f / y}$ Kras $^{G 12 D}$, Aifl/y WT ki Kras ${ }^{G 12 D}$, and Aiffly MT ki Kras ${ }^{G 12 D}$ mice. In line with the bioenergetic profiles, AlF-deficient cells harbored significantly less pyruvate and $\mathrm{NADH}$, but much more lactate (Fig. 7c), suggesting that the OXPHOS was indeed inhibited after AIF depletion. Importantly, intermediate metabolites belonging to the tricarboxylic acid cycle (TCA cycle), including citrate, malate, and succinate, were highly augmented in AIF-deficient cells (Fig. 7c), as further validated by Kyoto Encyclopedia of Genes and Genomes (KEGG) pathway enrichment analysis (Fig. 7d). All the metabolic alterations associated with Aif deficiency could be fully or partially restored by re-expressing WT or mitochondria-anchored AIF (Fig. 7c).

AIF is frequently overexpressed in human lung tumors and high AIF expression is associated with poor survival

Finally, we explored the clinical relevance of AIF in human lung cancer by retrospectively analyzing published datasets. Transcriptome studies of both lung adenocarcinoma (Okayama dataset; GEO: GSE31210) ${ }^{20,21}$ and different lung cancer subtypes (Rosseaux dataset; GEO: GSE30219) ${ }^{22}$ demonstrated a significant upregulation of the AIF mRNA levels in lung cancer tissue compared to normal tissue (Fig. 8a). To validate this finding at the protein level, we investigated AIF expression in human lung cancer samples by immunohistochemistry. In line with the previous findings, we observed elevated AIF expression in the tumor tissue from both
Kras-mutated and Kras WT lung cancer patients compared to their normal lung tissue (Fig. 8b). We did not detect significant differences in AIF expression among primary lung cancer, locally advanced lung cancer or metastatic lung cancer, which might be due to the limited number of patient samples with genetic workup as well as with long-term follow-up. Since AIF is an important component of respiration chain complex I, we then explored whether other components would show a similar upregulation in lung tumor tissue compared to normal lung tissue. We found that, in three different NSCLC cohorts (Okayama, Rosseaux and The Cancer Genome Atlas, TCGA), not only AIF but also the large majority of genes encoding the complex I subunits or their assembly factors were significantly overexpressed in NSCLC tissues compared to normal adjacent lung tissue (Fig. 8c). Accordingly, when we infected pneumocytes isolated from Aif $^{+/ y}$ and Aif ${ }^{+/ y}$ Kras $^{G 12 D}$ mice with Ad5-mSPC-Cre, a significant induction of AIF expression was detected 8 days after Kras ${ }^{G 12 D}$ activation; however, this effect was not observed in non-transformed pneumocytes expressing WT Kras (Fig. 8d). Furthermore, we evaluated the correlation between AIF expression and overall survival by analyzing RNAseq data from the TCGA dataset. High AIF expression was negatively correlated with poor prognosis, irrespective of the KRAS mutational status (Fig. 8e), suggesting that AIF overexpression is a common event in lung cancers associated with poor prognosis. In accord, patients bearing NSCLCs that express low levels of AIF protein, as determined by immunohistochemistry, exhibited a longer survival than patients bearing AIF ${ }^{\text {high }}$ tumors (Fig. 8f), supporting the conclusion that high AlF expression is associated with poor prognosis. 
a

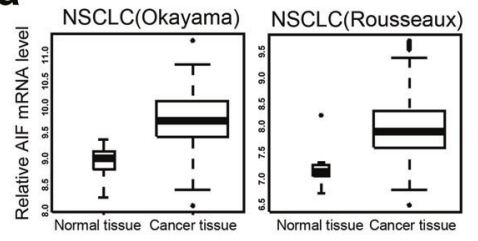

d

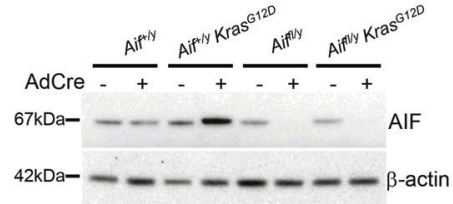

e

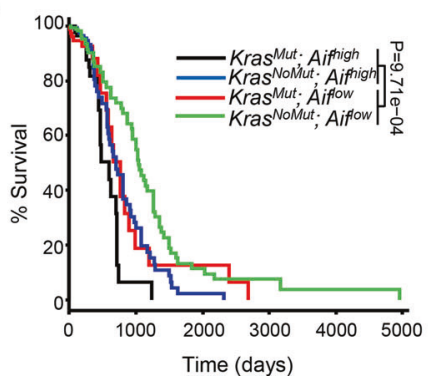

b
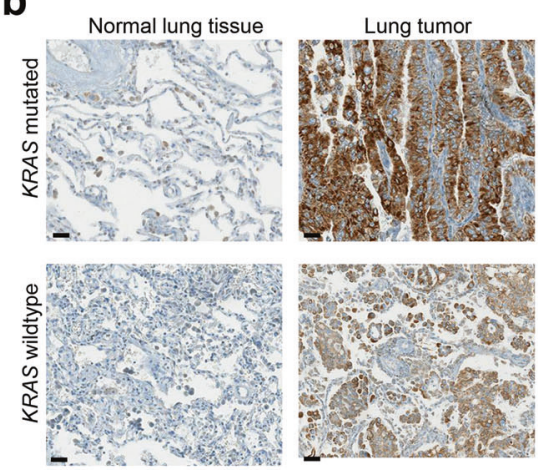

f

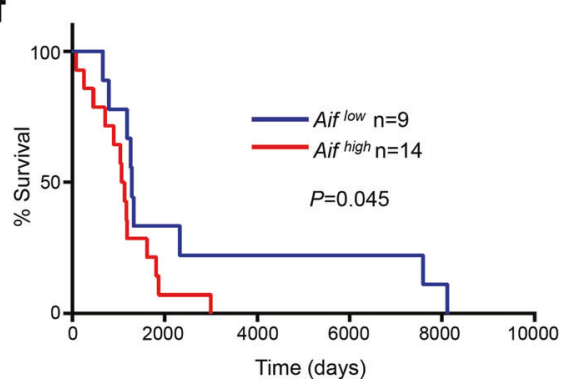

c

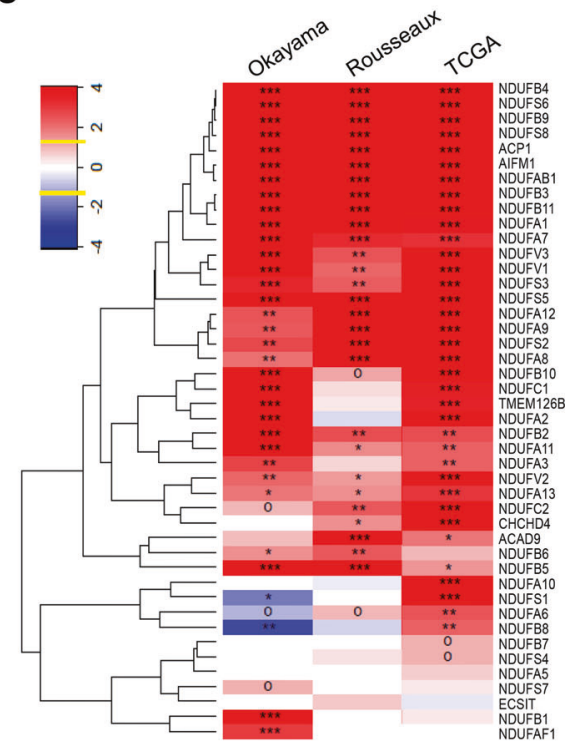

Fig. 8 AIF is frequently overexpressed in human lung tumors, and high AIF expression is associated with poor survival. a Comparison of AIF mRNA expression level between normal lung tissue and lung tumor tissue derived from NSCLC patients in two independent studies, namely Okayama dataset (GEO: GSE31210) and Rousseaux dataset (GEO: GSE30219). P-value was obtained by Student's t-test (linear model). b Representative AIF protein expression as determined by immunohistochemistry in lung tumors and tumor-adjacent normal lung tissue from KRAS-mutated and KRAS WT lung cancer patients. Scale bar, $20 \mu \mathrm{m}$. c Differential expression analysis of individual components from respiratory chain complex I, comparing tumor and normal lung tissues. Data were generated from the Okayama, Rousseaux and TCGA databases, respectively. Colors represent assigned $\log _{10}$ of $P$-values extracted from $t$-tests (red for overexpression, blue for underexpression in tumor compared to the adjacent normal tissue). Yellow lines in the color key (top left) represent the significance thresholds of \pm log 10 ( 0.05 ). Heatmap cells are annotated according to the statistical significance: ${ }^{* *} P<0.001,{ }^{* *} P<0.01,{ }^{*} P<0.05$, o $P<0.1$. d Immunoblot analysis of AIF expression in primary pneumocytes purified from Aif ${ }^{+/ y}$, Aif $^{+/ y} \mathrm{Kras}^{G 12 D}$, Aiffly , and Aif ${ }^{f / y} \mathrm{Kras}^{G 12 D}$ mice which were infected with or without Ad5-mSPC-Cre $(\mathrm{MOI}=100)$ for 8 days. $\beta$-actin is shown as a loading control. e Overall survival curves according to KRAS mutational status

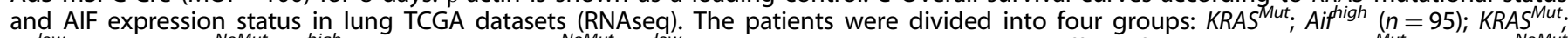

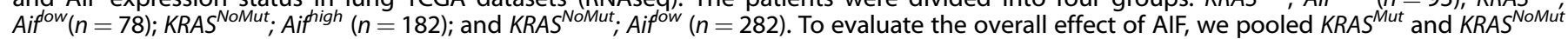
patients together and $P$ value indicated in the panel was obtained by applying a cox model. Patients were also stratified according to the $K R A S$ mutation status and evaluated by constructing a stratified cox model; $P=0.00673$ (KRAS $S^{\text {NoMut. Aif }}{ }^{\text {high }}$ vs KRAS ${ }^{\text {NoMut. }}$; Aif ${ }^{\text {Jow }}$ ) and $P=0.0438$ $\left(K_{R A S^{M u t}}\right.$; Aif ${ }^{\text {high }}$ vs KRAS ${ }^{\text {Mut }}$; Aif $\left.{ }^{\text {fow }}\right)$. f Overall survival curves stratified by AIF protein expression levels, as determined by immunohistochemistry. The patient cohort was divided into two groups: Aif $f^{\text {high }}(n=14)$ and Aif $f^{\text {OW }}(n=9)$. Due to the limited patient numbers, we pooled Kras ${ }^{\text {Mut }}$ and Kras ${ }^{\text {NoMut }}$ patients. $P=0.045$ (Log rank test)

\section{DISCUSSION}

Among the established hallmarks of cancer are a reduced propensity of the tumor cells to die as well as an altered metabolism, ${ }^{23}$ and these two characteristics may be intertwined in mechanistic terms. ${ }^{24}$ Resistance against cell death allows tumor cells to avoid elimination subsequent to the activation of cellintrinsic pathways or in response to a hostile microenvironment. Tumor cell metabolism is rewired to facilitate the generation of biomass and consequent proliferation. Based on these premises, we expected that elimination of AIF should have stimulated Kras ${ }^{G 12 D}$-driven lung oncogenesis because the absence of AIF would (i) render the cells resistant against (at least some) lethal stimuli and (ii) induce a Warburg-like metabolic reprogramming due to the partial inhibition of OXPHOS. In contrast to our expectations, however, AIF favored Kras ${ }^{G 12 D}$-driven carcinogenesis, and this effect can be attributed to the metabolic function of AIF, because a mitochondrion-retained, apoptosis-deficient mutant of AIF could replace WT AIF with respect to its protumorigenic activity.

One hallmark of cancer is the reprogramming of energy metabolism. Already 90 years ago, Otto Warburg presented evidence that cancer cells preferably use glycolysis for ATP production and produce lactate from glucose even under normoxic conditions. ${ }^{11}$ Therefore, one major therapeutic strategy for cancer treatment is to inhibit glycolysis in cancer cells and to promote OXPHOS, forcing the cell into a more "normal" metabolism which would presumably hinder cancer cell survival and growth. ${ }^{25}$ Drugs like 2-DG and lonidamine targeting the enzyme hexokinase, as well as Cap-232/TLN-232, an agent that targets the last step of glycolysis by inhibiting pyruvate kinase, are in clinical trials for a variety of solid tumors. ${ }^{26,27}$ Intriguingly, in our Kras $^{G 12 D}$-driven lung cancer model, the switch to glycolysis does not promote tumor growth and is disadvantageous for the cancer. These results are in accord with the fact that AIF expression is not lost in human cancer, irrespective of the Kras mutational status, probably reflecting the need for AIF to sustain the bioenergetics of malignant cells. Moreover, several recent studies have suggested that unlike many other cancers, lung tumors are highly oxidative and OXPHOS is indeed required for lung cancer development. ${ }^{28-30}$ Several specific inhibitors of OXPHOS including BAY 87-2243, ${ }^{31,32}$ IACS $-010759^{33,34}$ and VLX600 35 have been shown to mediate anticancer effects in suitable preclinical models. It is important to note that, in our study, OXPHOS was genetically modified in cancer cells only, while the aforementioned pharmacological studies cannot distinguish whether OXPHOS inhibition in neoplastic or stromal (including immune) cells accounts for the 
therapeutic effects of the inhibition of mitochondrial respiration. That said, cancer cell-specific AIF knockout only partially inhibited OXPHOS, meaning that it did not completely disrupt mitochondrial bioenergetics, yet yielded tangible effects on tumor incidence and progression.

Based on the aforementioned observations, it may be interesting to explore the possibility to inhibit AIF as well as the metabolic pathways that depend on it, in particular complex I of the respiratory chain ${ }^{36}$ with the scope of limiting malignant growth.

\section{MATERIALS AND METHODS}

Mice

Aiffoxed mice were generated by homologous recombination. ${ }^{3}$ Exon 7 of the Aif gene was targeted. These mice were backcrossed for at least 10 generations onto a C57/BI6 background and then crossed to $L S L-K r a s^{G 12 D 12}$ mice to generate Aif ${ }^{f / y} L S L-K r a s{ }^{G 12 D}$ and Aif $^{+/ y} L S L-K$ ras $^{G 12 D}$ littermate mice. WT AIF knock-in mice (AIF-3×FLAG; WT ki) and mitochondria-anchored AIF knock-in mice (AIF $\Delta$ 96-110/3× FLAG; MT ki) were generated using Rosa26 locus gene targeting. ${ }^{37}$ These lines were backcrossed 10 times onto the C57/Bl6 background and further crossed to Aif/y LSLKras $^{G 12 D}$ and Aif $^{+/ y}$ LSL-Kras ${ }^{G 12 D}$ mice (see breeding scheme in Supplementary information, Fig. S7a). Genotypes were determined by PCR. Only littermate mice were used in all experiments. All our mice were maintained according to ethical animal licenses complying with Austrian and European legislation.

\section{Induction of lung cancer}

Inhalation of 6-8-week-old mice with Ad5-CMV-Cre (VVC-U of lowa-5) or Ad5-mSPC-Cre (VVC-Berns-1168) viruses was performed as previously reported. ${ }^{38}$ All experimental animals were anaesthetized with $10 \%$ Ketasol/Xylasol and placed on a heated pad. An AdCre- $\mathrm{CaCl}_{2}$ precipitate was produced by mixing $60 \mu \mathrm{L} \mathrm{MEM}$, $2.5 \mu \mathrm{L}$ Adeno-Cre (10 ${ }^{10}$ p.f.u./mL; University of lowa, Gene Transfer Vector Core lowa, USA) and $0.6 \mu \mathrm{L} \mathrm{CaCl}_{2}(1 \mathrm{M})$ for each mouse and incubated for $20 \mathrm{~min}$ at room temperature.

\section{Histology and immunohistochemistry}

All lung tumors were analyzed histologically as previously reported. ${ }^{38}$ Briefly, lungs were cut into $2 \mu \mathrm{m}$ sections from at least 3 different planes and stained with haematoxylin and eosin. Lung sections were scanned using a Mirax slide scanner and lung/ tumor areas were scored by an algorithm programmed and executed using the Definiens software suite and visually controlled in a blinded way. For Ki67, cleaved caspase 3, AIF and SP-C immunoperoxidase staining, paraffin-embedded sections were dehydrated and antigenic epitopes were exposed using a 10-mM citrate buffer and microwaving. Sections were incubated with rabbit polyclonal anti-Ki67 (Novocastra), anti-active caspase 3 (Cell Signaling Technology, \#9661), anti-AIF (Abcam, ab32516), anti-PML (provided by G. Ferbeyre) and anti-SP-C (M20, Santa Cruz Biotechnology) antibodies. Primary Ab staining was detected by peroxidase-conjugated anti-rabbit IgG (DAKO, P0448, 1:500). Positive cells were counted on 15 randomly chosen tumor areas at $100 \times$ magnifications in a double-blinded fashion. Quantitative analysis was performed using HistoQuest ${ }^{\mathrm{Tm}}$ software (TissueGnostics $\mathrm{GmbH}$, Vienna, Austria, www.tissuegnostics.com).

\section{microCT scanning}

Formaldehyde-fixed lungs were stained in a solution of $1 \%$ elemental iodine and $2 \%$ potassium iodide in distilled water for 3 days. After staining, lung samples were rinsed and mounted in plastic tubes for microcomputed tomography (CT) scanning. Lung samples were scanned using a SCANCO $\mu$ CT 35 (SCANCO Medical AG, Brüttisellen, Switzerland) with a source energy of $70 \mathrm{keV}$ and an intensity of $114 \mu \mathrm{A}$ using a $0.2-\mathrm{mm}$ copper filter. Images were recorded with an angular increment of $0.36^{\circ}$. Reconstructed
microCT slices measured 1,024 $\times 1,024$ pixels (voxel size $=20 \mu \mathrm{m}$ ). Images were imported into Amira 5.3 (Visualization Sciences Group, Mérignac Cedex, France) and filtered with a threedimensional median filter $(3 \times 3 \times 3$ kernel). To discriminate background from lung tissue, an X-ray attenuation value of $\mu=0.3987$ and for lung tissue from tumor tissue an attenuation value of $\mu=1.2776$ were used. On the basis of segmentation, lung tissue and tumor volumes were calculated. ${ }^{39}$

Mitochondrial bioenergetics

Primary pneumocytes were purified and analyzed as described. ${ }^{38,40}$ Lungs were removed from 6-8-week-old mice, infiltrated with dispase $(2 \mu \mathrm{g} / \mathrm{mL})$ through the trachea, incubated for $30 \mathrm{~min}$ at room temperature and then manually minced and sequentially passed through $70 \mu \mathrm{m}$ and $35 \mu \mathrm{m}$ filters to obtain single-cell suspensions. Next, the cells were washed in minimum essential medium (a-MEM) and red blood cells were lysed using Red Blood Cell Lysis Buffer. Macrophages were removed by plating the cells on mouse $\operatorname{lgG}(0.5 \mathrm{mg} / \mathrm{mL})$-coated Petri dishes at $37^{\circ} \mathrm{C}$ for $30 \mathrm{~min}$ to $1 \mathrm{~h}$. Non-adherent cells were pelleted, resuspended in MEM supplemented with $10 \%$ FCS and plated again on $10 \mathrm{~cm}$ cell culture dishes for the removal of fibroblasts. Non-adherent pneumocytes were then plated on collagen-coated $6 \mathrm{~cm}$ dishes $(160 \mu \mathrm{g}$ per $6 \mathrm{~cm}$; Collagen Solution (Type I, Sigma) for $2 \mathrm{~h}$ at room temperature in Ham's F-12 media supplemented with $15 \mathrm{mM}$ HEPES, $0.8 \mathrm{mM} \mathrm{CaCl}_{2}, 0.25 \%$ BSA, ITS (Sigma) and 2\% BSA). Following purification, $2 \times 10^{5}$ cells were seeded on XF24 cell culture plates coated with collagen type I. Six replicates per cell type were assayed in each experiment using Seahorse technology (Seahorse Bioscience). Cells were incubated at $37^{\circ} \mathrm{C}$ in a $5 \% \mathrm{CO}_{2}$ incubator for $24 \mathrm{~h}$ until they were fully attached to the plate. On the day of the experiment, cells were washed and incubated in $675 \mu \mathrm{L}$ XF Assay media (8.3 g/L DMEM base, $3.7 \mathrm{~g} / \mathrm{L} \mathrm{NaCl}$, phenol red, $25 \mathrm{mM}$ glucose, $2 \mathrm{mM}$ L-glutamine, $\mathrm{pH} 7.4$ ) for $1 \mathrm{~h}$ at $37^{\circ} \mathrm{C}$. Oxygen consumption was measured under basal conditions in the presence of the mitochondrial inhibitors oligomycin $(0.5 \mu \mathrm{mol} / \mathrm{L}$, Calbiochem) or rotenone $(0.25 \mu \mathrm{mol} / \mathrm{L}, \mathrm{Sigma})$, or in the presence of the mitochondrial uncoupler FCCP $(0.3 \mu \mathrm{mol} / \mathrm{L}$, Sigma). Experiments were performed at $37^{\circ} \mathrm{C}$. OCR and ECAR were calculated by the oligomycin- or FCCP-induced changes in comparison to basal rates. The total protein of each well was determined by Bradford assay and used as the reference to normalize the OCR and ECAR.

Human lung cancer cell culture, clonogenic, and proliferation assay

NSCLC A549 and A427 cells were cultured with F-12 medium; $\mathrm{H} 1437, \mathrm{H} 1975, \mathrm{H} 460, \mathrm{H} 727, \mathrm{H} 1650$, and H358 cells were maintained in RPM1640 medium. The medium was supplemented with $10 \%$ fetal bovine serum (FBS) and $10 \mathrm{mM}$ HEPES buffer. The human lung tumor cells were infected with scrambled (SCR) shRNAs and different shRNAs targeting human AIF or CHCHD4 using the pGFP-C-shLenti vector (Origene). In case of AIF, the following two shRNA sequences were used:

(1) 5'-GCTGGAGCAGAGGTGAAGAGTAGAACAAC-3',

(2) 5'-CAGCCACCTTCTITCTATGTCTCTGCTCA-3'.

For CHCHD4 downregulation, the following three shRNAs were used:

(1) 5'-GGTACTACCACAGAGCTGGAGCTGAGGAA-3',

(2) 5'-CCATTGAGGCCACTGCAACCAAAGAAGAG-3',

(3) 5'-GAAGGATCGAATCATATTTGTAACCAAAG-3'.

Briefly, $5 \times 10^{4}$ cells/well were seeded $24 \mathrm{~h}$ prior to infection with the different lentiviral particles employing a multiplicity of infection (MOI) of 5-25, following the manufacturer's recommendations. To select infected cells, single cells were sorted into 96-well plates using FACS DIVA (Becton Dickinson, Franklin Lakes, $\mathrm{NJ}$, USA). For the assessment of clonogenic capacity, a single GFP ${ }^{+}$ cell was seeded in each well of 96 -well plates. Images of GFP ${ }^{+}$live cells were acquired with a Molecular Device ImageXpress 
Microscope 7-12 days after sorting. Quantifications of the numbers of clones and $\mathrm{GFP}^{+}$areas of clones were done with at least 96 sorted cells. To evaluate the cell proliferation, we selected stably infected clones by adding $1.5 \mu \mathrm{g} / \mathrm{mL}$ puromycin to the cell cultures. Stable clones were obtained by single cell sorting as above mentioned for $\mathrm{GFP}^{+}$live cells selection. Cells stably expressing control shRNAs (SCR), AIF shRNAs (shAIF) and CHCHD4 shRNAs (shCHCHD4) were seeded in 96-well imaging plates (Greiner Bio-One, Kremsmünster, Austria) at densities of 500, 1000 and 2000 cells/well. Images of $\mathrm{GFP}^{+}$live cells were acquired with a Molecular Device ImageXpress Microscope at the $0,24,48$, and $72 \mathrm{~h}$ timepoints. Nuclei were counterstained using Hoechst $33342(1 \mu \mathrm{M})$.

Clones used for proliferation assay were transfected with the plasmids pBUDneo ( $p B U D$ ) or pBUDneo-MLS-CHCHD4 ( $p B U D$ $\mathrm{CHCHD} 4)^{8}$ by means of Lipofectamine $2000^{\mathrm{TM}}$ transfection reagent (Invitrogen, Carlsbad, CA, USA). Selection of transfected cells was achieved by adding $1 \mathrm{mg} / \mathrm{mL}$ geneticin and the selected cells were further maintained in $750 \mu \mathrm{g} / \mathrm{mL}$ geneticine. All cell cultures were kept at $37{ }^{\circ} \mathrm{C}$ in a humidified incubator under $5 \% \mathrm{CO}_{2}$ atmosphere. Unless stated otherwise, media and supplements for cell culture were purchased from Thermo Fisher Scientific (Carlsbad, CA, USA).

\section{Determination of $\mathrm{pH}$, lactate, and ATP levels}

The cell culture medium was collected on days $0-3$ and the $\mathrm{pH}$ was measured using a $\mathrm{pH}$ meter (Hana Instruments). For the measurements of lactate levels, the culture medium was collected and adherent cells were trypsinized and counted using a counting chamber. Lactate amount in media was determined by measuring oxidized 2,6-dichlorophenol-indophenol (DCPIP), which is reduced by phenazine methosulfate (PMS); in turn PMS is reduced by NADH produced by lactate dehydrogenase (LDH) that oxidizes lactate to pyruvate at the same time. DCPIP oxidation was spectrophotometrically measured at $600 \mathrm{~nm}$. Intracellular ATP levels were measured with the Bioluminescence Assay Kit HSII (Roche). The intensity of bioluminescence can be correlated with the amount of ATP in a sample. Pneumocytes were trypsinized after 36-h incubation in culture media with glucose $(5 \mathrm{~g} / \mathrm{L})$, without glucose or with glucose plus 2-DG (concentration of 0-6 mM). According to the manufacturer's protocol, the cells were washed once in PBS, resuspended in dilution buffer and incubated for $5 \mathrm{~min}$ with the provided lysis buffer. Luciferin was added in the luminometre and subsequently measured by bioluminescence. A standard curve was established using several dilutions of the ATP standard provided with the kit.

\section{Cytofluorometry}

To assess apoptosis and cell viability, pneumocytes were trypsinized and stained with $\mathrm{PI}(5 \mu \mathrm{g} / \mathrm{mL})$ or 4 ',6-diamino-2phenylindole-dihydrochloride (DAPI, $5 \mu \mathrm{g} / \mathrm{mL}$ ) and incubated at $37^{\circ} \mathrm{C}$ for $20 \mathrm{~min}$, followed by cytofluorimetric analysis.

\section{Western blotting}

Western blotting was performed following standard protocols. The following primary antibodies reactive to mouse AIF (Abcam, ab32156, 1:1000), Complex I NDUFB6 (Abcam, ab110244, 1:1000), Complex I NDUFS3 (Abcam, ab110246, 1:1000), Complex I NDUFA9 (Abcam, ab14713, 1:1000), and $\beta$-actin (Sigma, F3022, 1:10,000) were used. For western blotting of human lung cancer cell lysates, we used primary antibodies specific for AIFM1 (human AIF, $4642 \mathrm{~S}$, Cell Signaling Technology), CHCHD4 (HPA034688, Prestige Antibodies, Sigma-Aldrich), MitoProfile Total OXPHOS Human WB Antibody Cocktail (ab110411, Abcam) containing 5 mAbs against Complex I subunit NDUFB8 (ab110242), NDUFS7 (ab105025), NDUFA12 (ab192617), Complex II subunit SDHB (ab14714), Complex III subunit UQCRC2 (ab14745), Complex IV subunit II (ab110258), and ATP synthase subunit a as a marker for complex V (ab14748), and $\beta$-actin-HRP (mouse monoclonal, AC-15, ab49900, Abcam) as a loading control. Membranes were incubated overnight with the primary antibodies at $4{ }^{\circ} \mathrm{C}$ (diluted in $2.5 \% \mathrm{BSA}$ in $1 \times$ TBST buffer), washed three times in TBST for 15 min each and probed with horseradish peroxidase-conjugated secondary Abs (1:5000, Promega) at room temperature for $1 \mathrm{~h}$. Ab binding was visualized by enhanced chemiluminescence (GE Healthcare, RPN2106). When needed, membranes were incubated in stripping solution Restore ${ }^{\mathrm{TM}}$ PLUS Western Blot Stripping Buffer for $10 \mathrm{~min}$ and re-blotted according to the manufacturer's instructions. Densitometry was conducted employing ImageJ and using $\beta$-actin to normalize for protein expression.

Determination of metabolites

Cell pellets $\left(10^{6}\right.$ cells) were extracted using a MeOH:ACN:H2O $(2: 2: 1, \mathrm{v} / \mathrm{v})$ solvent mixture. A volume of $1 \mathrm{~mL}$ of cold solvent was added to each pellet, vortexed for $30 \mathrm{~s}$, and incubated in liquid nitrogen for $1 \mathrm{~min}$. The samples were then allowed to thaw at room temperature and sonicated for $10 \mathrm{~min}$. This cycle of cell lysis in liquid nitrogen combined with sonication was repeated three times. To precipitate proteins, the samples were incubated for $1 \mathrm{~h}$ at $-20^{\circ} \mathrm{C}$, followed by centrifugation at $13,000 \mathrm{rpm}$ for $15 \mathrm{~min}$ at $4{ }^{\circ} \mathrm{C}$. The resulting supernatant was removed and evaporated to dryness in a vacuum concentrator. The dry extracts were then reconstituted in $100 \mu \mathrm{L}$ of $\mathrm{ACN}: \mathrm{H} 2 \mathrm{O}(1: 1, \mathrm{v} / \mathrm{v})$, sonicated for $10 \mathrm{~min}$, and centrifuged at $13,000 \mathrm{rpm}$ for $15 \mathrm{~min}$ at $4{ }^{\circ} \mathrm{C}$ to remove insoluble debris. The supernatants were transferred to Eppendorf tubes, shock frozen and stored at $-80^{\circ} \mathrm{C}$ prior to LC/MS analysis. One microliter of the metabolite extract was injected on a ZICpHILIC HPLC column operated at a flow rate of $100 \mu \mathrm{L} / \mathrm{min}$, directly coupled to a TSQ Quantiva mass spectrometer (Thermo Fisher Scientific). The following transitions were used for quantitation in the negative ion mode: AMP $346 \mathrm{~m} / z$ to $79 \mathrm{~m} / \mathrm{z}$, ADP $426 \mathrm{~m} / z$ to $134 \mathrm{~m} / \mathrm{z}_{\text {, ATP }} 506 \mathrm{~m} / \mathrm{z}$ to $159 \mathrm{~m} / \mathrm{z}$, IMP $347 \mathrm{~m} / \mathrm{z}$ to $79 \mathrm{~m} / \mathrm{z}$, GMP $362 \mathrm{~m} / \mathrm{z}$ to $211 \mathrm{~m} / \mathrm{z}$, GDP $442 \mathrm{~m} / \mathrm{z}$ to $344 \mathrm{~m} / \mathrm{z}$, GTP 522 $\mathrm{m} / \mathrm{z}$ to $424 \mathrm{~m} / \mathrm{z}$, taurine $124 \mathrm{~m} / \mathrm{z}$ to $80 \mathrm{~m} / \mathrm{z}$, malate $133 \mathrm{~m} / \mathrm{z}$ to 115 $\mathrm{m} / \mathrm{z}$, citrate $191 \mathrm{~m} / \mathrm{z}$ to $111 \mathrm{~m} / \mathrm{z}$, pyruvate $87 \mathrm{~m} / \mathrm{z}$ to $43 \mathrm{~m} / \mathrm{z}$, lactate $89 \mathrm{~m} / z$ to $43 \mathrm{~m} / \mathrm{z}$, NADH $664 \mathrm{~m} / \mathrm{z}$ to $408 \mathrm{~m} / \mathrm{z}$, NAD $662 \mathrm{~m} / \mathrm{z}$ to 540 $\mathrm{m} / \mathrm{z}$, hexose phosphates $259 \mathrm{~m} / \mathrm{z}$ to $97 \mathrm{~m} / \mathrm{z}$, Acetyl CoA $808 \mathrm{~m} / \mathrm{z}$ to $408 \mathrm{~m} / \mathrm{z}$, CoA $766 \mathrm{~m} / \mathrm{z}$ to $408 \mathrm{~m} / \mathrm{z}$, succinate $117 \mathrm{~m} / \mathrm{z}$ to $73 \mathrm{~m} / \mathrm{z}$. Glutamine $147 \mathrm{~m} / \mathrm{z}$ to $130 \mathrm{~m} / \mathrm{z}$, glutamate $148 \mathrm{~m} / \mathrm{z}$ to $84 \mathrm{~m} / \mathrm{z}$, serine $106 \mathrm{~m} / \mathrm{z}$ to $60 \mathrm{~m} / \mathrm{z}$ were measured in the positive ion mode. For all transitions, the optimal collision energy was defined by analyzing pure metabolite standards. Chromatograms were manually interpreted using trace finder (Thermo Fisher Scientific), validating experimental retention times with the respective quality controls. All measurements were within the linear range of detection.

For the metabolomics analysis, the metabolite concentration was normalized using a Z-score normalization method with the formula of $y=(x-a) / \lambda$, in which $x$ refers to the real concentration, $a$ indicates the mean value of all samples, and $\lambda$ is the variance of all samples. The normalized concentrations of metabolites were applied to generate a heatmap, which showed the concentration difference of all metabolites. For KEGG (http://www.kegg.jp, Tokyo, Japan) pathway analysis, the clusterProfiler $\mathrm{R}$ package was employed.

\section{D tumor spheroid cultures}

A flat round drop of Matrigel (Corning) was seeded in cell culture plates followed by incubation at $37^{\circ} \mathrm{C}$ for $5 \mathrm{~min}$. Primary lung tumor cells were mixed with the Matrigel and kept on ice until they were seeded onto the droplet of Matrigel in the plate in "a droplet on a droplet" fashion. The Matrigel plug was incubated at $37{ }^{\circ} \mathrm{C}$ for $30 \mathrm{~min}$ and then covered with cell culture medium. Images were acquired and analyzed 7 days later. For BrdU staining, tumor spheroids were incubated with $10 \mu \mathrm{M} / \mathrm{mL}$ BrdU at $37^{\circ} \mathrm{C}$ for $2 \mathrm{~h}$, followed by $3.7 \%$ formaldehyde fixation at room temperature for $15 \mathrm{~min}$. Spheres were then permeabilized with 
PBST for 20 min and treated with $1 \mathrm{M} \mathrm{HCl}$ and $2 \mathrm{M} \mathrm{HCl}$, followed by neutralization with a phosphate/citric acid buffer. Tumor spheroids were then stained with an anti-BrdU antibody (Abcam, ab6326) at $4{ }^{\circ} \mathrm{C}$ overnight, visualized ${ }^{20}$ with a fluorescence secondary antibody, and counterstained with DAPI.

Human cohort studies

The selected datasets used in this study are: Lung-Okayama (GSE31210), ${ }^{20,21}$ Lung-Rousseaux (GSE30219) ${ }^{22}$ and Lung Cancer RNA-seq from TCGA (https://cancergenome.nih.gov/). For the TCGA dataset, we extracted the KRAS mutation data and the clinical survival data; mRNA counts were normalized by using the "voom" algorithm of the limma package. ${ }^{41}$ For differential expression between lung tumor and normal lung tissues, $t$-tests were performed. For survival analysis, Cox model tests were performed. Human immunohistochemistry (IHC) analysis was conducted in lung tumors as well as the healthy tissue adjacent to the lung tumors using a monoclonal antibody against human AIF (Abcam, ab32516). IHC staining was quantified using $\mathrm{H}$-scores (range 0-300), which incorporate staining intensity (range 0-3) and the percentage of positively-stained tumor cells (range $0-100 \%)$. The distribution of AIF expression was analyzed by the square root scale method. AIF ${ }^{\text {high }}$ and AIF $^{\text {low }}$ samples were defined as 20 percentiles of the highest or lowest AIF expressors; survival is presented in Kaplan Meier curves (log rank test).

\section{Statistics}

Normally distributed data was statistically analyzed using unpaired two-tailed $t$-tests for single comparisons, and two-way analysis of variance (ANOVA) for multiple comparisons. ANOVA analyses were followed by Bonferroni's post hoc tests. Ordinary data was analyzed using the unpaired two-tailed Mann-Whitney test. Overall survival was analyzed in Kaplan-Meier curves using a log-rank test. Microscopy images were segmented and analyzed by means of the MetaXpress (Molecular Devices) software and numerical data were further processed with R software (http:// www.r-project.org/). Unless otherwise specified, data are presented as means \pm SEM. The statistical tests and $P$ values are indicated in each figure legend. $P \leq 0.05$ was considered to indicate statistical significance. Numbers of mice per group used in each experiment are annotated in the corresponding figure legends as $n$. All the scripts used for the analysis are available upon reasonable request.

\section{ACKNOWLEDGEMENTS}

We would like to thank all members of our laboratories for helpful discussions. J.M.P. is supported by grants from IMBA, the Austrian Ministry of Sciences, the Austrian Academy of Sciences, an ERC Advanced Grant, and an Era of Hope Innovator award. J.J.H. is supported by NH \& MRC project grants 1047995, 1051485, and 1104441. G.K. is supported by the Ligue contre le Cancer (équipe labellisée); Agence National de la Recherche (ANR) - Projets blancs; ANR under the frame of E-Rare-2, the ERA-Net for Research on Rare Diseases; Association pour la recherche sur le cancer (ARC); Cancéropôle lle-de-France; Chancelerie des universités de Paris (Legs Poix), Fondation pour la Recherche Médicale (FRM); a donation by Elior; European Research Area Network on Cardiovascular Diseases (ERA-CVD, MINOTAUR); Gustave Roussy Odyssea, the European Union Horizon 2020 Project Oncobiome; Fondation Carrefour; Highend Foreign Expert Program in China (GDW20171100085 and GDW20181100051), Institut National du Cancer (INCa); Inserm (HTE); Institut Universitaire de France; LeDucq Foundation; the LabEx Immuno-Oncology; the RHU Torino Lumière; the Seerave Foundation; the SIRIC Stratified Oncology Cell DNA Repair and Tumor Immune Elimination (SOCRATE); and the SIRIC Cancer Research and Personalized Medicine (CARPEM).

\section{AUTHOR CONTRIBUTIONS}

S.R., L.M. and B.P. with help from D.S., I.U., I.K. and S.J.C. performed and analyzed the lung tumor experiments. T.H. and R.H. generated the inducible mutant, mitochondrial-anchored, and WT AIF constructs which were used by K.H. and J.J.H. to generate the knock-in Aif mice. L.M., V.S., N.M., M.L. and O.K. characterized AIF in human cells. L.K. performed certified pathology analysis. A.J., A.L. and A.B. performed Seahorse analysis. P.Z. performed metabolites analysis with coordination from Z.S.; G.S. analyzed human relevance study together with F.Q. and H.P.; G.K. and J.M.P. conceived the study and wrote the paper with the help of S.R.

\section{ADDITIONAL INFORMATION}

Supplementary information accompanies this paper at https://doi.org/10.1038/ s41422-019-0181-4.

Competing interests: The authors declare no competing interests.

\section{REFERENCES}

1. Susin, S. et al. Molecular characterization of mitochondrial apoptosis-inducing factor. Nature 397, 441-446 (1999).

2. Vanessa, P. M. et al. Downregulation of apoptosis-inducing factor in Harlequin mutant mice sensitizes the myocardium to oxidative stress-related cell death and pressure overload-induced decompensation. Circ. Res. 96, e92-e101 (2005).

3. Joza, N. et al. Muscle-specific loss of apoptosis-inducing factor leads to mitochondrial dysfunction, skeletal muscle atrophy, and dilated cardiomyopathy. Mol. Cell Biol. 25, 10261-10272 (2005).

4. Schulthess, F. T. et al. Deletion of the mitochondrial flavoprotein apoptosis inducing factor (AIF) induces b-cell apoptosis and impairs b-cell mass. PLoS One 4, e4394 (2009).

5. Vahsen, N. et al. AIF deficiency compromises oxidative phosphorylation. EMBO J. 23, 4679-4689 (2004).

6. Pospisilik, J. A. et al. Targeted deletion of AIF decreases mitochondrial oxidative phosphorylation and protects from obesity and diabetes. Cell 131, 476-491 (2007).

7. Cheung, E. C. C. et al. Dissociating the dual roles of apoptosis-inducing factor in maintaining mitochondrial structure and apoptosis. EMBO J. 25, 4061-4073 (2006).

8. Hangen, E. et al. Interaction between AIF and CHCHD4 regulates respiratory chain biogenesis. Mol. Cell 58, 1001-1014 (2015).

9. Cairns, R. A., Harris, I. S. \& Mak, T. W. Regulation of cancer cell metabolism. Nat. Rev. Cancer 11, 85-95 (2011).

10. Palsson-Mcdermott, E. M. \& O'Neill, L. A. J. The Warburg effect then and now: from cancer to inflammatory diseases. Bioessays 35, 965-973 (2013).

11. Warburg, O. On the origin of cancer cells. Science 123, 309-314 (1956).

12. Johnson, L. et al. Somatic activation of the K-ras oncogene causes early onset lung cancer in mice. Nature 410, 1111-1116 (2001).

13. Jackson, E. L. et al. Analysis of lung tumor initiation and progression using conditional expression of oncogenic K-ras. Genes Dev. 15, 3243-3248 (2001).

14. Nikitin, A. Y. et al. Classification of proliferative pulmonary lesions of the mouse: recommendations of the mouse models of human cancers consortium. Cancer Res. 64, 2307-2316 (2004).

15. Vernier, M. et al. Regulation of E2Fs and senescence by PML nuclear bodies. Genes Dev. 25, 41-50 (2011).

16. Gesta, S. et al. Mesodermal developmental gene Tbx15 impairs adipocyte differentiation and mitochondrial respiration. Proc. Natl Acad. Sci. USA 108, 2771-2776 (2011)

17. Otera, H., Ohsakaya, S., Nagaura, Z.-I., Ishihara, N. \& Mihara, K. Export of mitochondrial AIF in response to proapoptotic stimuli depends on processing at the intermembrane space. EMBO J. 24, 1375-1386 (2005).

18. Brown, D. et al. Loss of Aif function causes cell death in the mouse embryo, but the temporal progression of patterning is normal. Proc. Natl Acad. Sci. USA 103, 9918-9923 (2006).

19. Tammela, T. et al. A Wnt-producing niche drives proliferative potential and progression in lung adenocarcinoma. Nature 545, 355-359 (2017).

20. Okayama, H. et al. Identification of genes upregulated in ALK-positive and EGFR/KRAS/ALK-negative lung adenocarcinomas. Cancer Res. 72, 100-112 (2012).

21. Yamauchi, M., Yamaguchi, R., Nakata, A., Kohno, T. \& Nagasaki, M. Epidermal growth factor receptor tyrosine kinase defines critical prognostic genes of Stage I lung adenocarcinoma. PLoS One 7, e43923 (2012).

22. Rousseaux, S. et al. Ectopic activation of germline and placental genes identifies aggressive metastasis-prone lung cancers. Sci. Transl. Med. 5, 186 ra66 (2013).

23. Hanahan, D. \& Weinberg, R. A. Hallmarks of cancer: the next generation. Cell 144, 646-674 (2011).

24. Green, D. R., Galluzzi, L. \& Kroemer, G. Cell biology. Metabolic control of cell death. Science 345, 1250256 (2014).

25. Fogg, V. C., Lanning, N. J. \& MacKeigan, J. P. Mitochondria in cancer: At the crossroads of life and death. Chin. J. Cancer 30, 526-539 (2011). 
26. Tennant, Da, Durán, R. V. \& Gottlieb, E. Targeting metabolic transformation for cancer therapy. Nat. Rev. Cancer 10, 267-277 (2010).

27. Madhok, B. M., Yeluri, S., Perry, S. L., Hughes, Ta \& Jayne, D. G. Dichloroacetate induces apoptosis and cell-cycle arrest in colorectal cancer cells. Br. J. Cancer 102, 1746-1752 (2010).

28. Doucette, S. et al. Lactate metabolism in human lung tumors. Cell 171, 358-371 (2017).

29. Hensley, C. T. et al. Metabolic heterogeneity in human lung tumors. Cell 164, 681-694 (2016).

30. Jacks, T. et al. Environment impacts the metabolic dependencies of Ras-driven non-small cell lung cancer. Cell Metab. 23, 517-528 (2016).

31. Ellinghaus, P. et al. BAY $87-2243$, a highly potent and selective inhibitor of hypoxia-induced gene activation has antitumor activities by inhibition of mitochondrial complex I. Cancer Med. 5, 611-624 (2013).

32. Schöckel, L. et al. Targeting mitochondrial complex I using BAY 87-2243 reduces melanoma tumor growth. Cancer Metab. 3, 11 (2015).

33. Molina, J. R. et al. An inhibitor of oxidative phosphorylation exploits cancer vulnerability. Nat. Med. 24, 1036-1046 (2018).

34. Vangapandu, H. V. et al. Biological and metabolic effects of IACS-010759, an OxPhos inhibitor, on chronic lymphocytic leukemia cells. Oncotarget 9 24980-24991 (2018).

35. Zhang, X. et al. Induction of mitochondrial dysfunction as a strategy for targeting tumour cells in metabolically compromised microenvironments. Nat. Commun. 5, 3295 (2015)

36. Birsoy, K. et al. Metabolic determinants of cancer cell sensitivity to glucose limitation and biguanides. Nature 508, 108-112 (2014).
37. Nyabi, O. et al. Efficient mouse transgenesis using Gateway-compatible ROSA26 locus targeting vectors and F1 hybrid ES cells. Nucleic Acids Res. 37, e55 (2009).

38. Schramek, D. et al. The stress kinase MKK7 couples oncogenic stress to p53 stability and tumor suppression. Nat. Genet. 43, 212-219 (2011).

39. Metscher, B. D. MicroCT for developmental biology: a versatile tool for highcontrast 3D imaging at histological resolutions. Dev. Dyn. 238, 632-640 (2009).

40. Rao, S. et al. A dual role for autophagy in a murine model of lung cancer. Nat. Commun. 5, 3056 (2014).

41. Ritchie, M. E. et al. Limma powers differential expression analyses for RNA-sequencing and microarray studies. Nucleic Acids Res. 43, e47 (2015).

(i) Open Access This article is licensed under a Creative Commons Attribution 4.0 International License, which permits use, sharing, adaptation, distribution and reproduction in any medium or format, as long as you give appropriate credit to the original author(s) and the source, provide a link to the Creative Commons license, and indicate if changes were made. The images or other third party material in this article are included in the article's Creative Commons license, unless indicated otherwise in a credit line to the material. If material is not included in the article's Creative Commons license and your intended use is not permitted by statutory regulation or exceeds the permitted use, you will need to obtain permission directly from the copyright holder. To view a copy of this license, visit http://creativecommons. org/licenses/by/4.0/.

(c) The Author(s) 2019 